\title{
Global Attractor of Nonlocal Nonlinear Schrödinger Equation on R
}

\author{
Chaosheng Zhu \\ School of mathematics and statistics, Southwest University, Chongqing, 400715, P. R. China. \\ Email: zcs@swu.edu.cn
}

\begin{abstract}
This paper is devoted to the large time behavior and especially to the regularity of the global attractor of the dissipative 1D nonlinear Schrödinger equation with nonlocal integral term on $\mathbf{R}$. We first prove that the existence of the global attractor $\mathcal{A}_{\gamma}$ in the strong topology of $H^{1}(\mathbf{R})$ and the existence of the exponential attractor $\mathcal{M}$ which contains the global attractor $\mathcal{A}_{\gamma}$, are still finite dimensional, and attract the trajectories exponentially fast. We also show that the global attractor $\mathcal{A}_{\gamma}$ is regular, i.e., $\mathcal{A}_{\gamma}$ is included, bounded and compact in $H^{2}(\mathbf{R})$ assuming that the forcing term $f(x)$ is of class $H^{2}(\mathbf{R})$. Furthermore we estimate the number of the determining modes for this equation. Moreover, we show that the solution trajectories and the global attractor of the nonlocal Schrödinger equation converge to those of the usual Schrödinger equation, as the coefficient of the nonlocal integral term goes to zero.
\end{abstract}

Keywords: Nonlinear Schrödinger equation, Nonlocal integral term, Global attractor, Exponential attractor, Regularity, Determining modes, Trajectory convergence, Attractor convergence.

\section{Introduction}

This paper is devoted to the large time behavior and especially to the regularity of the global attractor of the following dissipative 1D nonlinear Schrödinger (NLS) equation with nonlocal integral term on $\mathbf{R}$ :

$$
\begin{gathered}
i u_{t}+u_{x x}+|u|^{2} u+\gamma u \int_{-\infty}^{x}|u|^{2} d x+i \alpha u=f(x), t \in(0,+\infty), x \in \mathbf{R}, \\
u(x, 0)=u_{0}(x), \quad x \in \mathbf{R},
\end{gathered}
$$

where the damping parameter $\alpha>0$ and the dispersion parameter $\gamma \in \mathbf{R}$, the unknown function $u=u(x ; t)$ is complex and the complex function $f(x) ; x \in \mathbf{R}$ is known. Throughout this paper, let $\bar{u}$ is the complex conjugate of $u,|u|^{2}=u \bar{u}$. Set $\|u\|^{2}=:\|u\|_{L^{2}(\mathbf{R})}^{2}=\int_{\mathbf{R}}|u|^{2} d x,\|u\|_{H^{1}(\mathbf{R})}^{2}=\|u\|^{2}+\left\|u_{x}\right\|^{2}$, $\|u\|_{H^{2}(\mathbf{R})}^{2}=\|u\|^{2}+\left\|u_{x}\right\|^{2}+\left\|u_{x x}\right\|^{2}$, and $H^{-1}(\mathbf{R})$ is the dual space of $H^{1}(\mathbf{R})$.

Equation (1.1) models the influence of ion inertia upon the nonlinear Langmuir waves on the basis of the Zakharov equations that perturbs the classical NLS equation

$$
i u_{t}+u_{x x}+|u|^{2} u+i \alpha u=f .
$$

The long time behavior of solutions to (1.3) has been studied in many papers. For example, in [44], the existence of global attractor for NLS was obtained by energy equation. In [15], the existence of the global attractor was established and the finite dimensionality of the attractor for NLS was proved. It was also proved that the global attractor is regular provided that the function $f(x)$ is smooth enough. On the other hand, the regularity of the global attractor was proved in [17] for the one dimensional case with periodic boundary conditions. This result improved that of [15] where the existence of a global attractor for the weak topology in $H^{1}([0,1])$ was proved (among other results). In the one dimensional case, it was also proved in [17] that the attractor is made of $C^{\infty}([0,1])$ functions if the external force $f(x)$ is $C^{\infty}([0,1])$. This result was recently improved in [38], where the authors proved that the global attractor is included in Gevrey space if the external force $f(x)$ belongs to some Gevrey space.

Equation (1.1) was first studied by [30] which gives the derivation of the nonlocal integral term with $f=\alpha=0$. For equation (1.1), Huang has proved in [29] that there also exists a compact global attractor 
in a special weighted space. Later, Ma and Chang [33] studied the semi-dicretized NLS equation (1.1) and proved that for each mesh size, there exist attractors for the discretized system. In the one-dimensional case for Equation (1.1), it is also proved in [47] that the attractor is made of $H^{2}([0,1])$ functions if the external force $f(x)$ is $H^{2}([0,1])$. In this paper, our main aim is to improve results in [47]. To this end, we first prove that the existence of the global attractor $\mathcal{A}_{\gamma}$ in the strong topology of $H^{1}(\mathbf{R})$ and the existence of the exponential attractor $\mathcal{M}$ which contains the global attractor $\mathcal{A}_{\gamma}$, are still finite dimensional, and attract the trajectories exponentially fast. Secondly, we prove that the global attractor $\mathcal{A}_{\gamma}$ is regular, i.e., $\mathcal{A}_{\gamma}$ is included, bounded and compact in $H^{2}(\mathbf{R})$ assuming that $f(x)$ is of class $H^{2}(\mathbf{R})$. Furthermore we estimate the number of the determining modes for this equation. Moreover, we show that the solution trajectories and the global attractor of the nonlocal Schrödinger equation converge to those of the usual Schrödinger equation, as the coefficient of the nonlocal integral term goes to zero.

It is well known that the existence of the global attractor for dissipative evolution equations has always relied on some kind of compactness of the semigroup generated by such equations. Usually, this compactness is obtained through some regularization property of such equations together with the compact imbedding of the relevant Sobolev spaces (see [42] for instance). This approach is only suitable for bounded domains, the Sobolev imbeddings are no longer compact otherwise. For unbounded domains, the remedy was to consider weighted spaces (see F. Abergel [1,2], A. V. Babin [3], A. V. Babin and M. I. Vishik [6], and E. Feireisl et al [11]), but with the drawback that the forcing term and in some cases even the initial condition had to be restricted to the weighted spaces. Similarly, for equation (1.1), Huang has proved in [29] that there also exists a compact global attractor in a special weighted space, provided the effect of the zero-order dissipation is large enough.

In section 2, our aim is to avoid weighted spaces by methods in [12,31] and by the so-called Kuratowski $\alpha$-measure of noncompactness to prove the asymptotical smoothness of $S_{\gamma}(t)$. The idea of using the Kuratowski $\alpha$-measure to obtain the existence of the global attractor was successfully applied to weakly damped Klein-Gordon-Schrödinger equations by B. L. Guo and Y. S. Li [20]. For that purpose, we are going to establish time-uniform a priori estimates of solutions $u$ in $L^{2}(\mathbf{R})$, next in $H^{1}(\mathbf{R})$ and in $H^{2}(\mathbf{R})$. Then we apply these estimates to obtain the existence of the global solutions and bounded absorbing sets in $H^{1}(\mathbf{R})$. Next we shall split $S_{\gamma}(t)$ so that we can make use of Kuratowski $\alpha$-measure of noncompactness to prove the asymptotical smoothness of $S_{\gamma}(t)$. Thus, by the theory in [22], we can prove the following theorem about existence of maximal compact attractor.

Theorem 1.1 Let $f \in H^{1}(\mathbf{R}), S_{\gamma}(t)$ be the semigroup generated by (1.1)-(1.2). Then there exists a set $\mathcal{A}_{\gamma} \subset H^{1}(\mathbf{R})$ satisfying:

(I) $S_{\gamma}(t) \mathcal{A}_{\gamma}=\mathcal{A}_{\gamma}, \forall t \geq 0$;

(II) $\lim _{t \rightarrow \infty} \operatorname{dist}_{H^{1}(\mathbf{R})}\left(S_{\gamma}(t) B, \mathcal{A}_{\gamma}\right)=\lim _{t \rightarrow \infty} \sup _{y \in B} \operatorname{dist}_{H^{1}(\mathbf{R})}\left(S_{\gamma}(t) B, \mathcal{A}_{\gamma}\right)=0, \forall B \subset H^{2}(\mathbf{R})$ bounded;

(III) $\mathcal{A}_{\gamma}$ is compact in $H^{1}(\mathbf{R})$. That is, $\mathcal{A}_{\gamma}$ is a maximal compact attractor in $H^{1}(\mathbf{R})$ which attracts bounded sets of $H^{2}(\mathbf{R})$ in the topology of $H^{1}(\mathbf{R})$.

Here, it is worth mentioning that in [45], the authors introduced a new concept, called the norm-to-weak continuous semigroup in a Banach space, and gave a technical theorem to verify this notion of continuity. Then they established a general method which is necessary and sufficient to obtain the existence of the global attractor for this kind of semigroup. By this method, we improved this result in our another paper.

In section 3, we shall prove the existence of exponential attractor. It follows from the previous literature (see $[22,42]$ for instance) that it is not always possible to embed the global attractor into a proper smooth finite dimensional manifold. Nevertheless, and also in view of the possible defaults of the global attractor as discussed in the introduction, it can be useful to construct larger (not necessarily smooth) sets which contain the global attractor and are still finite dimensional, and attract the trajectories exponentially fast. This led Eden, Foias, Nicolaenko, and Temam to propose the notation of an exponential attractor (also sometime called an inertial set) in [10]. In this section, we shall prove the following theorem about the existence of exponential attractor.

Theorem 1.2 There exists a positively invariant subset $B$ of $H^{1}(\mathbf{R})$ such that $\mathcal{A}_{\gamma} \subset B$ and the dynamical system $\left(\left\{S_{\gamma}(t)\right\}_{t \geq 0}, B, H^{1}(\mathbf{R})\right)$ admits an exponential attractor $\mathcal{M}$. 
In section 4 , we shall prove that the global attractor $\mathcal{A}_{\gamma}$ is regular. For dissipative evolutionary partial differential equations, which enjoy asymptotic smoothness (also called asymptotic compactness) properties, the set $\mathcal{J}$ of all globally defined and bounded solutions for $t \in \mathbf{R}$, plays a special role [39]. In general, the elements in this set $\mathcal{J}$ should enjoy certain regularity properties in space and the trajectories in $\mathcal{J}$ should be as smooth in the time variable as the nonlinearity of the equation. We point out that, in the autonomous case, under additional dissipation hypotheses, this set $\mathcal{J}$ coincides with the compact global attractor of the equation. These spaces or time regularity properties of $\mathcal{J}$ are obviously true for equations, which are smooth in finite time, such as ordinary differential equations or semi-linear parabolic equations [27]. For example, the Navier-Stokes equations have this property of smoothness in finite time. When the system is not smooth in finite time, regularity (in space or time) of the elements $\mathcal{J}$ can be very difficult to prove or could be even false. Note that regularity results are primordial in the theory of perturbations of invariant sets and in particular of periodic orbits, as shown in [24] (see also [25]). Numerous authors have shown regularity properties for $\mathcal{J}$ in the case of dynamical systems which are not smoothness in finite time. For retarded functional differential equations in $\mathbf{R}^{n}$ with finite delay or neutral functional differential equations, such results were already obtained thirty years ago by Hale [21], Lopes [32], Nussbaum [37]. For dissipative evolutionary equations, which admit a compact global attractor, regularity results have been proved by several authors, using different methods (for the earliest results, see, for example, [16] for the damped wave equation, [17,18,38] for the weakly damped Schrödinger equations, [35] for the weakly damped, forced Korteweg de Vries equation, and [41] for a review). In [16], in a same argument, Ghidaglia and Temam have shown space and time regularity in $C^{k}$-type spaces for the global attractor of the damped wave equation (from their proof, one could not deduce analyticity neither in time, nor in the spatial variables). In [17], Goubet showed the existence of the compact global attractor and its regularity in $H^{k}([0,1])$-spaces for the one-dimensional weakly Schrödinger wave equation by using a Galerkin method. Applying the same Galerkin method, Oliver and Titi [38] have shown that this compact global attractor belongs actually to a Gevrey regularity class. By the same method, Wang [43] obtained the regularity results for global attractor of Benjamin-Bona-Mahony equation in a bounded interval $\Omega \subset \mathbf{R}$.

Here we follow a similar strategy, however, our proofs are more complex because the problems (1.1)(1.2) are studied on unbounded domain $\mathbf{R}$ and because of the presence of the non-local nonlinear term $\gamma u \int_{-\infty}^{x}|u|^{2} d x$. We introduce a splitting of the high-frequency part of the solution $u$ of (1.1)-(1.2) into a regular part and a small one that converges towards 0 when $t$ goes to infinity, and we devote to the existence result for the regular part. We also study the long time behavior of the difference between the high frequency part of $u$ and its regular part. In order to prove the regularity of global attractor, we have to impose some assumption on the force $f$, that is $f(x) \in H^{2}(\mathbf{R})$. Then, we apply those results to complete the proofs of the following theorem.

Theorem 1.3 If $f(x)$ is $H^{2}(\mathbf{R})$ complex-valued functions, then the global attractor $\mathcal{A}_{\gamma}$ is included, bounded and compact in $H^{2}(\mathbf{R})$.

In section 5, we shall deduce the so-called "finite number of determining modes property" for the system (1.1)-(1.2). The property of "finite number of determining modes" was introduced and proved for the two-dimensional Navier-Stokes equations by Foias and Prodi in 1967 [14] and generalized by C. Foias, O. Manley, R. Temam and Y. Treve [13]. It was shown for the two-dimensional Navier-Stokes equations that the large time behavior of the orbits is completely determined by its projection on a spectral infinte-dimensional space. This result was subsequently extended to numerous (strongly) dissipative equations. In [23], Hale and Raugel introduced the Galerkin method to obtain determining modes property for the general frame of semilinear equations. Following Goubet [17] we want to show that the orbits on global attractor $\mathcal{A}_{\gamma}$ are fully determined by their projections $P_{N}$, where $N$ is sufficiently large. This property tells that the asymptotic behavior in terms of (1.1)-(1.2) depends only on a finite number of determining modes.

Theorem 1.4 Let $\left\{u_{1}(t)\right\}$ and $\left\{u_{2}(t)\right\}$ be two complete orbits of $\mathcal{A}_{\gamma}$ such that $P_{N} u_{1}(t)=P_{N} u_{2}(t)$, for all $t \in \mathbf{R}$, and $N \geq N_{0}, N_{0}$ as in Lemma 4.1 below. Then $u_{1}(t)=u_{2}(t)$, for all $t \in \mathbf{R}$. 
We point out that we also could directly prove Theorem 1.4, without applying Theorem 1.3, by performing appropriate a priori estimates. But, showing Theorem 1.4 as a consequence of Theorem 1.3 is much shorter.

In section 6 , we will show that individual solution trajectories and the global attractor for the nonlocal Schrödinger equation (1.1) converge to the solution trajectories and the global attractor of the usual Schrödinger equation (1.3), as the coefficient $\gamma$ of the nonlocal integral term goes to zero. General convergence result for the global attractor is available in Hale et al. [22], Temam [42] and Hale, Lin and Raugel [26]. Similar applications can also be found in Hill and Sulin [28]. Let us denote by $S_{\gamma}(t)$ and $S(t)$ the solution operator for the nonlocal Schrödinger equation (1.1), and the usual Schrödinger equation (1.3), respectively. We need to verify a few conditions that will ensure the convergence of the solution trajectories and attractors. In particular, we should show that operators $S_{\gamma}(t)$ and $S(t)$ satisfy

$$
\left\|S_{\gamma}(t) u_{0}-S(t) u_{0}\right\|_{H^{1}(\mathbf{R})} \leq \Gamma\left(\gamma, t, u_{0}\right)
$$

where $\Gamma\left(\gamma, t, u_{0}\right) \rightarrow 0$ as $\gamma \rightarrow 0$, and that the domain of attraction of $S_{\gamma}(t)$ is independent of $\gamma$. For more details of these conditions see Theorem I.1.2 of Temam [42], or Theorem 2.4 of Hale, Lin and Raugel [26].

Theorem 1.5 When the coefficient $\gamma$ goes to zero, a solution trajectory of (1.1) converges in $H^{1}(\mathbf{R})$ to a solution trajectory of (1.3), as long as both trajectories start at the same initial point. Furthermore, the global attractor of (1.1) converges in $H^{1}(\mathbf{R})$ to the global attractor of (1.3).

This paper is organized as follows. In Section 2, by the Kuratowski $\alpha$-measure of noncompactness, we prove the existence of maximal compact attractor. In Section 3, we shall prove the existence of exponential attractor. In Section 4, we shall prove that the global attractor $\mathcal{A}_{\gamma}$ is regular. In Section 5, we show that the orbits on global attractor $\mathcal{A}$ are fully determined by their projections $P_{N}$, where $N$ is sufficiently large. In Section 6, we will show that individual solution trajectories and the global attractor for the nonlocal Schrödinger equation (1.1) converge to the solution trajectories and the global attractor of the usual Schrödinger equation (1.3), as the coefficient $\gamma$ of the nonlocal integral term goes to zero.

\section{$2 \quad$ Proof of Theorem 1.1}

In this section, we shall prove the existence of global attractor for the problems (1.1)-(1.2). To this end, let us start with the following lemmas:

Lemma 2.1 [29] ( $i)$ Let $f \in L^{2}(\mathbf{R})$. Then for every $u_{0} \in H^{1}(\mathbf{R})$, there exists a unique solution $u(t) \in C\left((0,+\infty), H^{1}(\mathbf{R})\right) \cap C^{1}\left((0,+\infty), H^{-1}(\mathbf{R})\right)$ to (1.1)-(1.2). Moreover, the solution operator $S_{\gamma}(t)$ : $u_{0} \mapsto u(t)$ is continuous from $H^{1}(\mathbf{R})$ onto itself and has a bounded absorbing set

$$
B=\left\{u \mid u \in H^{1}(\mathbf{R}),\|u\|_{H^{1}(\mathbf{R})} \leq \rho\right\} \subset H^{1}(\mathbf{R}) .
$$

(ii) Let $f \in L^{2}(\mathbf{R})$. Then for every $u_{0} \in H^{2}(\mathbf{R})$, there exists a unique solution $u(t) \in C\left((0,+\infty), H^{2}(\mathbf{R})\right) \cap$ $C^{1}\left((0,+\infty), L^{2}(\mathbf{R})\right)$ to (1.1)-(1.2). Moreover, the solution operator $S_{\gamma}(t): u_{0} \mapsto u(t)$ is continuous from $H^{2}(\mathbf{R})$ onto itself and has a bounded absorbing set $B_{2} \subset H^{2}(\mathbf{R})$.

If $f(x) \in L^{2}(\mathbf{R})$ is independent of $t$, then $S_{\gamma}(t)$ forms a semigroup. In order to prove the existence of global attractor, we have to impose some assumption on the force $f$, that is $f(x) \in H^{1}(\mathbf{R})$. Let $B \subset H^{1}(\mathbf{R})$ be a bounded set, then $S_{\gamma}(t) B \subset H^{1}(\mathbf{R})$ is also bounded. In this section we shall split $S_{\gamma}(t)$ so that we can make use of the so-called Kuratowskii $\alpha$-measure of noncompactness to prove the asymptotic smoothness of $S_{\gamma}(t)$. More precisely, we split $S_{\gamma}(t)$ into two parts: $S_{1}(t)$ and $S_{2}(t)$, with $\alpha\left(S_{1}(t) B\right) \rightarrow 0$ as $t \rightarrow \infty$ and $S_{2}(t) B$ is relatively compact in $H^{1}(\mathbf{R})$, where the $\alpha$-measure of a set $A \subset H^{1}(\mathbf{R})$ is defined by $\alpha(A) \equiv \inf \{d \mid$ there is a finite covering of $A$ of diameter $<d\}$. Then

$$
\alpha\left(S_{\gamma}(t) B\right) \leq \alpha\left(S_{1}(t) B\right)+\alpha\left(S_{2}(t) B\right)=\alpha\left(S_{1}(t) B\right) \rightarrow 0 \text { as } t \rightarrow \infty .
$$


Let $B \subset H^{1}(\mathbf{R})$ with $\sup _{u \in B}\|u\|_{H^{2}(\mathbf{R})} \leq R, u(t)=S_{\gamma}(t) u_{0}$ be the solution of (1.1)- (1.2) with initial data $u_{0} \in B$. Recall that $u(t)$ is uniformly bounded in $H^{2}(\mathbf{R})$. For $L>0$, we consider a $C^{\infty}$-smooth cut-off function $\chi_{L}$ such that $0 \leq \chi_{L} \leq 1$ and

$$
\chi_{L}(x)=\left\{\begin{array}{l}
1, \text { if }|x| \leq L, \\
0, \text { if }|x| \geq 1+L .
\end{array}\right.
$$

Since $f \in H^{1}(\mathbf{R})$, the sequence $\left(f \chi_{L}\right)$ converges to $f$ in $H^{1}(\mathbf{R})$ as $L \rightarrow+\infty$. Thus, for any $\eta \in(0,1)$, there exists $L(\eta)>0$ such that

$$
\left\|f-f_{\eta}\right\|_{H^{1}(\mathbf{R})} \leq \eta, \text { where } f_{\eta}=f \chi_{L(\eta)}
$$

Since $H^{2}(\mathbf{R})$ is continuously embedded in $L^{\infty}(\mathbf{R})$, thus $u \in C\left([0,+\infty), L^{\infty}(\mathbf{R})\right), u_{x x} \in C\left([0,+\infty), L^{2}(\mathbf{R})\right)$ and the nonlinear terms $|u|^{2} u \in C\left([0,+\infty), L^{\infty}(\mathbf{R})\right), u \int_{-\infty}^{x}|u|^{2} d x \in C\left([0,+\infty), L^{\infty}(\mathbf{R})\right)$. We then infer from linear semigroup theory [40] that the problem:

$$
\begin{array}{r}
i v_{\eta t}+(1-i \eta) v_{\eta x x}+|u|^{2} v_{\eta}+\gamma v_{\eta} \int_{-\infty}^{x}|u|^{2} d x+i \alpha v_{\eta}=f-f_{\eta}-i \eta u_{x x}, \\
v_{\eta}(x, 0)=u_{0}(x),
\end{array}
$$

has a unique solution $v_{\eta} \in C^{1}\left((0,+\infty), L^{2}(\mathbf{R})\right) \cap L_{l o c}^{2}\left((0,+\infty), H^{1}(\mathbf{R})\right)$. We define a function $w_{\eta}$ of $C^{1}\left((0,+\infty), L^{2}(\mathbf{R})\right)$ by $w_{\eta}=u-v_{\eta}$, then $w_{\eta}$ satisfies the following equation:

$$
\begin{array}{r}
i w_{\eta t}+(1-i \eta) w_{\eta x x}+|u|^{2} w_{\eta}+\gamma w_{\eta} \int_{-\infty}^{x}|u|^{2} d x+i \alpha w_{\eta}=f_{\eta}, \\
w_{\eta}(x, 0)=0 .
\end{array}
$$

Next we shall prove the following two lemmas.

Lemma 2.2 There exists a function $\tau(\eta):(0,1) \rightarrow[0,+\infty)$ and a constant $k_{1}>0$ depending only on $\gamma$, $f$ such that, for any $\eta \in(0,1)$ and $u_{0} \in H^{2}(\mathbf{R})$,

$$
\begin{gathered}
\left\|v_{\eta}\right\|_{H^{1}(\mathbf{R})} \leq k_{1}, \quad t \geq 0, \\
\left\|v_{\eta}\right\|_{H^{1}(\mathbf{R})} \leq k_{1} \sqrt{\eta}, t \geq \tau(\eta) .
\end{gathered}
$$

Proof. Multiplying (2.3) by $\bar{v}_{\eta}$, taking the imaginary part, and integrating on $\mathbf{R}$, we obtain

$$
\frac{d}{d t}\left\|v_{\eta}\right\|^{2}+2 \eta\left\|v_{\eta x}\right\|^{2}+2 \alpha\left\|v_{\eta}\right\|^{2} \leq 2\left\|f-f_{\eta}\right\|\left\|v_{\eta}\right\|+2 \eta\left\|u_{x}\right\|\left\|v_{\eta x}\right\| .
$$

It follows from Lemma 2.1 that $\left\|u_{x}\right\|<\rho_{1}$ for any $t \geq 0$. Thus, using also (2.2), we come to:

$$
\frac{d}{d t}\left\|v_{\eta}\right\|^{2}+2 \eta\left\|v_{\eta x}\right\|^{2}+2 \alpha\left\|v_{\eta}\right\|^{2} \leq 2 \eta\left\|v_{\eta}\right\|+2 \eta \rho_{1}\left\|v_{\eta x}\right\|
$$

hence $\frac{d}{d t}\left\|v_{\eta}\right\|^{2}+\alpha\left\|v_{\eta}\right\|^{2} \leq\left(\rho_{1}^{2}+\frac{1}{\gamma}\right) \eta$. By Gronwall inequality,

$$
\left\|v_{\eta}\right\|^{2} \leq\left\|u_{0}\right\|^{2} e^{-\alpha t}+\left(\rho_{1}^{2}+\frac{1}{\gamma}\right) \frac{\eta}{\alpha}\left(1-e^{-\alpha t}\right) .
$$

On the other hand, multiplying $(2.3)$ by $\bar{v}_{\eta x x}$, taking the imaginary part, and integrating on $\mathbf{R}$, we obtain

$$
\begin{aligned}
& \frac{d}{d t}\left\|v_{\eta x}\right\|^{2}+2 \eta\left\|v_{\eta x x}\right\|^{2}+2 \alpha\left\|v_{\eta x}\right\|^{2} \\
\leq & 2\left(\left\|f-f_{\eta}\right\|+\eta\left\|u_{x x}\right\|+\gamma\|u\|^{2}\left\|v_{\eta}\right\|+\|u\|_{L^{\infty}}^{2}\left\|v_{\eta}\right\|\right)\left\|v_{\eta x x}\right\| .
\end{aligned}
$$

Thus, $\frac{d}{d t}\left\|v_{\eta x}\right\|^{2}+\alpha\left\|v_{\eta x}\right\|^{2} \leq \eta C_{7}$, by Gronwall inequality,

$$
\left\|v_{\eta x}\right\|^{2} \leq\left\|u_{0 x}\right\|^{2} e^{-\alpha t}+\frac{\eta C_{7}}{\alpha}\left(1-e^{-\alpha t}\right) .
$$

Then, (2.7) and (2.8) follows at once from (2.10), the proof of Lemma 2.2 is completed. 
Lemma 2.3 For any $\eta \in(0,1)$, there exists a constant $k_{2}(\eta)>0$ depending only on $\gamma$, $f$ and $\eta$ such that, for any $u_{0} \in H^{2}(\mathbf{R})$,

$$
\left\|x w_{\eta}\right\|_{H^{1}(\mathbf{R})} \leq k_{2}(\eta), t \geq 0 .
$$

Proof. We infer from Lemma 2.1 and Lemma 2.2 that $\|u(t)\|_{H^{1}(\mathbf{R})} \leq \rho_{1},\left\|v_{\eta}(t)\right\|_{H^{1}(\mathbf{R})} \leq k_{1}, t \geq 0$. Multiplying (2.5) by $x^{2} \bar{w}_{\eta}$, taking the imaginary part, and integrating on $\mathbf{R}$, we obtain

$$
\frac{d}{d t}\left\|x w_{\eta}\right\|^{2}+2 \alpha\left\|x w_{\eta}\right\|^{2}+2 \eta\left\|x w_{\eta x}\right\|^{2} \leq 4(1+\eta)\left\|x w_{\eta x}\right\|\left\|w_{\eta}\right\|+2 \int_{\mathbf{R}} x^{2}\left|f_{\eta} w_{\eta}\right| d x .
$$

Since $f_{\eta}$ has compact support, $|x| f_{\eta} \in L^{2}(\mathbf{R})$,

$$
\frac{d}{d t}\left\|x w_{\eta}\right\|^{2}+2 \alpha\left\|x w_{\eta}\right\|^{2} \leq 4\left(\eta+\frac{1}{\eta}\right)\|\| w_{\eta}\|+2\| x w_{\eta}\|\| x f_{\eta} \|,
$$

then

Gronwall lemma then yields

$$
\frac{d}{d t}\left\|x w_{\eta}\right\|^{2}+\alpha\left\|x w_{\eta}\right\|^{2} \leq C_{8}\left(1+\frac{1}{\eta}+\left\|x f_{\eta}\right\|^{2}\right) .
$$

$$
\left\|x w_{\eta}\right\|^{2} \leq C_{9}\left(1+\frac{1}{\eta}+\left\|x f_{\eta}\right\|^{2}\right), \quad t \geq 0 .
$$

Now let us differentiate $(2.5)$ with respect to $x$, this leads to

$$
\begin{array}{r}
i w_{\eta x t}+(1-i \eta) w_{\eta x x x}+\left(|u|^{2}\right)_{x} w_{\eta}+|u|^{2} w_{\eta x} \\
+\gamma|u|^{2} w_{\eta}+\gamma w_{\eta x} \int_{-\infty}^{x}|u|^{2} d x+i \alpha w_{\eta x}=f_{\eta x}, \\
w_{\eta x}(x, 0)=0,
\end{array}
$$

Multiplying (2.13) by $x^{2} \bar{w}_{\eta x}$, taking the imaginary part, and integrating on $\mathbf{R}$, we obtain

$$
\begin{aligned}
& \frac{d}{d t}\left\|x w_{\eta x}\right\|^{2}+2 \alpha\left\|x w_{\eta x}\right\|^{2}+2 \eta\left\|x w_{\eta x x}\right\|^{2} \\
& =4 \int_{\mathbf{R}} x \operatorname{Im}\left(\bar{w}_{\eta x} w_{\eta x x}\right) d x-4 \eta \int_{\mathbf{R}} x \operatorname{Re}\left(\bar{w}_{\eta x} w_{\eta x x}\right) d x \\
& -2 \operatorname{Im} \int_{\mathbf{R}}\left(\left(|u|^{2}\right)_{x}+\gamma|u|^{2}\right) w_{\eta} x^{2} \bar{w}_{\eta x} d x+2 \int_{\mathbf{R}} x^{2} \operatorname{Im}\left(f_{\eta x} \bar{w}_{\eta x}\right) d x .
\end{aligned}
$$

By Young inequality and (2.12) we get

$$
\frac{d}{d t}\left\|x w_{\eta x}\right\|^{2}+\alpha\left\|x w_{\eta x}\right\|^{2} \leq C_{10}\left(1+\frac{1}{\eta}+\left\|x f_{\eta x}\right\|^{2}\right) .
$$

Gronwall lemma then yields

$$
\left\|x w_{\eta x}\right\|^{2} \leq C_{10}\left(1+\frac{1}{\eta}+\left\|x f_{\eta x}\right\|^{2}\right), \quad t \geq 0 .
$$

The proof of Lemma 2.3 is completed.

Now we are going to prove the Theorem 1.1. To this end, we need the following compact imbedding Lemma.

Lemma 2.4 ([20]) Let $s>s_{1}$ be integers. Then the imbedding of $H^{s}\left(\mathbf{R}^{n}\right) \cap H^{s_{1}}\left(\mathbf{R}^{n},\left(1+|x|^{2}\right) d x\right)$ into $H^{s_{1}}\left(\mathbf{R}^{n}\right)$ is compact.

Proof of Theorem 1.1. From Lemmas 2.3 and 2.4 we see that $S_{2}(t)$ defined by (2.5)-(2.6) is compact from $H^{2}(\mathbf{R})$ into $H^{1}(\mathbf{R})$. Therefore for any $B \subset H^{2}(\mathbf{R})$ bounded, $\alpha\left(S_{2}(t) B\right)=0, \forall t \geq 0$. From Lemma 2.2, we find that $\forall \varepsilon>0$, there exist an $\eta$ and thus a $t_{0}>0$ such that $\left\|S_{1}(t) u_{0}\right\| \leq \varepsilon, \forall t \geq t_{0}$, and $u_{0} \in B, B \subset H^{2}(\mathbf{R})$ bounded. That is, for such $\eta>0, \alpha\left(S_{1}(t) B\right) \leq 2 \varepsilon$, as $t \geq t_{0}$, so we have $\alpha\left(S_{\gamma}(t) B\right) \leq \alpha\left(S_{1}(t) B\right)+\alpha\left(S_{2}(t) B\right)=\alpha\left(S_{1}(t) B\right) \leq 2 \varepsilon$, as $t \geq t_{0}$. Hence $\lim _{t \rightarrow \infty} \alpha\left(S_{\gamma}(t) B\right)=0$. Therefore $S_{\gamma}(t)$ is asymptotically smooth. By the theory in [22] we complete the proof of Theorem 1.1. 


\section{Proof of Theorem 1.2}

In this section, we shall prove the existence of exponential attractor for the problems (1.1)-(1.2). To this end, let us start with the following definitions and lemmas:

Definition([5,7]) A compact set $\mathcal{M} \subset X$ is an exponential attractor for $S(t)$ if (i) it has finite fractal dimension, $\operatorname{dim}_{F} \mathcal{M}<+\infty$; (ii) it is positively invariant, $S(t) \mathcal{M} \subset \mathcal{M}$, for every $t \in[0,+\infty)$; (iii) it attracts exponentially the bounded subsets of $X$ in the following sense: $\forall B \subset X$ bounded, $\operatorname{dist}(S(t) B, \mathcal{M}) \leq Q\left(\|B\|_{X}\right) e^{-\alpha t}, t \in[0,+\infty)$, where the positive constant $\alpha$ and the monotonic function $Q$ are independent of $B$.

It follows from this definition that an exponential attractor, if it exists, contains the global attractor. The existence of exponential attractors relies on a dichotomy principle, called the squeezing property. Because of its importance, we recall its definition.

Definition([10,34]) Let $X$ be a subset of a Hilbert space $\mathcal{H}$; let $S$ be a mapping from $X$ to $X$, $S X \subset X$. We say that $S$ has squeezing property in $X$ if for some $\delta \in\left(0, \frac{1}{4}\right)$, there exists an orthogonal projection $P=P(\delta)$ of rank equal to $N_{0}(\delta)$ such that for every $u$ and $v$ in $X$ either $\|(I-P)(S u-S v)\|_{\mathcal{H}} \leq$ $\|P(S u-S v)\|_{\mathcal{H}}$, or $\|S u-S v\|_{\mathcal{H}} \leq \delta\|u-v\|_{\mathcal{H}}$.

We can note that this property makes an essential use of orthogonal projectors with finite rank, so the corresponding construction is valid in Hilbert spaces only.

Theorem 3.1 ([5]) Let $X$ be a closed invariant set in a Hilbert space $\mathcal{H}$ such that there exists a covering of $X$ by a finite number of balls of radius 1. Let the semigroup $\{S(t)\}$ have a global attractor on $\mathcal{H}$. Let for a fixed $t>0, S(t)$ have a squeezing property and be uniformly Lipschitzian on $X$. Then $\{S(t)\}$ has an exponential attractor on $X$.

Now, we take $\mathcal{H}=H^{1}(\mathbf{R})$ and choose $X=B$ as in (2.4). Using Theorem 3.1 we are able to prove the existence of exponential attractor. That is, the proof of Theorem 1.2 is based on the following three lemmas, that is, Lemma 3.2-3.4 below.

Lemma 3.2 There exists an invariant set $B \subset H^{1}(\mathbf{R})$ which can be covered by a finite number of unite balls.

The proof of Lemma 3.2 is standard (See [31]), so we omit it.

Lemma 3.3 The operators $\left\{S_{\gamma}(t)\right\}$ are Lipschitzian on $B$ for any $t \geq 0$ (the Lipschitz constant depends on $t$ ).

According to the definition of the mapping $S_{\gamma}(t), S_{\gamma}(t) u_{0}-S_{\gamma}(t) v_{0}=u(t)-v(t)$, where $u(t)$ and $v(t)$ are solutions of (1.1), with initial value $u_{0}$ and $v_{0}$, respectively. Subtracting the equations for $u$ and $v$, we obtain the equation

$$
\begin{gathered}
i w_{t}+w_{x x}+i \alpha w+\left(|u|^{2}+|v|^{2}\right) w+u v \bar{w} \\
+\gamma w \int_{-\infty}^{x}|u|^{2} d x+\gamma v \int_{-\infty}^{x}(u \bar{w}+w \bar{v}) d x=0, t>0, x \in \mathbf{R}, \\
w(x, 0)=u_{0}(x)-v_{0}(x), \quad x \in \mathbf{R},
\end{gathered}
$$

Proof. Multiplying (3.1) by $\bar{w}$, taking the imaginary part, and integrating on $\mathbf{R}$, we obtain

$$
\begin{aligned}
\frac{d}{d t}\|w\|^{2}+2 \alpha\|w\|^{2} & =-2 \operatorname{Im} \int_{\mathbf{R}} u v \bar{w}^{2} d x-2 \gamma \operatorname{Im} \int_{\mathbf{R}} v\left[\int_{-\infty}^{x}(u \bar{w}+w \bar{v}) d x\right] \bar{w} d x \\
& \leq 2\|u\|_{L^{\infty}(\mathbf{R})}\|v\|_{L^{\infty}(\mathbf{R})}\|w\|^{2}+2 \gamma(\|u\|+\|v\|)\|v\|\|w\|^{2}
\end{aligned}
$$


Hence, $\frac{d}{d t}\|w\|^{2} \leq C\|w\|^{2}$, by Gronwall inequality, we have $\|w(t)\|^{2} \leq e^{C t}$. Now multiplying (3.1) by $\bar{w}_{x x}$, taking the imaginary part, and integrating on $\mathbf{R}$, we obtain

$$
\begin{aligned}
\frac{d}{d t}\left\|w_{x}\right\|^{2}+2 \alpha\left\|w_{x}\right\|^{2} & =-2 \operatorname{Im} \int_{\mathbf{R}} u v \bar{w}^{2} d x-2 \gamma \operatorname{Im} \int_{\mathbf{R}} v\left[\int_{-\infty}^{x}(u \bar{w}+w \bar{v}) d x\right] \bar{w} d x \\
& \leq 2\|u\|_{L^{\infty}(\mathbf{R})}\|v\|_{L^{\infty}(\mathbf{R})}\|w\|^{2}+2 \gamma(\|u\|+\|v\|)\|v\|\|w\|^{2} .
\end{aligned}
$$

Hence, $\frac{d}{d t}\|w\|^{2} \leq C\|w\|^{2}$, by Gronwall inequality, we have $\|w(t)\|^{2} \leq e^{C t}$. The proof of Lemma 3.3 is completed.

Lemma 3.4 For any $\delta>0$ there exists such $t>0$ and $N$ that the operators $\left\{S_{\gamma}(t)\right\}$ have a squeezing property on $B$ in the space $H^{1}(\mathbf{R})$.

Now our main goal is the proof of Lemma 3.4. The proof of Lemma 3.4 is based on the next three technical lemmas, that is Lemma 3.5-3.7 below. For $L>0$, we consider a $C^{\infty}$-smooth cut-off function $\chi_{L}$ such that $0 \leq \chi_{L} \leq 1$ and

$$
\chi_{L}(x)=\left\{\begin{array}{l}
1, \text { if }|x| \leq L \\
0, \text { if }|x| \geq 1+L
\end{array}\right.
$$

Since $u \in H^{1}(\mathbf{R})$, the sequence $\left(u \chi_{L}\right)$ converges to $u$ in $H^{1}(\mathbf{R})$ as $L \rightarrow+\infty$. Thus, for any $\eta \in(0,1)$, there exists $L(\eta)>0$ such that

$$
\begin{aligned}
\left\|\left(1-\chi_{L}\right) u v\right\|_{H^{1}(\mathbf{R})} & \leq \eta, \\
\left\|v-v_{\eta}\right\|_{H^{1}(\mathbf{R})} & \leq \eta,
\end{aligned}
$$

Together with (3.1)-(3.2) we consider the equation

$$
\begin{gathered}
i \Phi_{t}+(1-i \eta) \Phi_{x x}+i \alpha \Phi+\left(|u|^{2}+|v|^{2}\right)\left(1-\chi_{L}\right) \Phi+u v\left(1-\chi_{L}\right) \bar{\Phi} \\
+\gamma \Phi\left(1-\chi_{L}\right) \int_{-\infty}^{x}|u|^{2} d x+\gamma v\left(1-\chi_{L}\right) \int_{-\infty}^{x}(u \bar{\Phi}+\Phi \bar{v}) d x+i \eta w_{x x}=0, t>0, x \in \mathbf{R} \\
\Phi(x, 0)=w(x, 0)=u_{0}(x)-v_{0}(x), \quad x \in \mathbf{R} .
\end{gathered}
$$

Let $\Psi=w-\Phi$. Obviously, $\Psi$ satisfies the equation

$$
\begin{array}{r}
i \Psi_{t}+(1-i \eta) \Psi_{x x}+i \alpha \Psi+\left(|u|^{2}+|v|^{2}\right) \Psi+u v \bar{\Psi}+\gamma \Psi \int_{-\infty}^{x}|u|^{2} d x \\
+\gamma v \int_{-\infty}^{x}(u \bar{\Psi}+\Psi \bar{v}) d x+\left(|u|^{2}+|v|^{2}\right) \chi_{L} \Phi+\gamma \Phi \chi_{L} \int_{-\infty}^{x}|u|^{2} d x \\
+u v \chi_{L} \bar{\Phi}+\gamma v \chi_{L} \int_{-\infty}^{x}(u \bar{\Phi}+\Phi \bar{v}) d x=0, \quad t>0, x \in \mathbf{R}, \\
\Psi(x, 0)=0, \quad x \in \mathbf{R} .
\end{array}
$$

Next we shall prove the following three lemmas.

Lemma 3.5 There exists a constant $k_{1}>0$ such that, for $\eta \in(0,1)$ small sufficiently and $w(x, 0) \in$ $H^{1}(\mathbf{R})$,

$$
\|\Phi\|_{H^{1}(\mathbf{R})} \leq k_{1}, t \geq 0 .
$$

Proof. Multiplying (3.6) by $\bar{\Phi}$, taking the imaginary part, and integrating on $\mathbf{R}$, we obtain

$$
\begin{aligned}
& \frac{d}{d t}\|\Phi\|^{2}+2 \eta\left\|\Phi_{x}\right\|^{2}+2 \alpha\|\Phi\|^{2}=2 \operatorname{Im} \int_{\mathbf{R}} i \eta w_{x} \bar{\Phi}_{x} d x \\
& -2 \operatorname{Im} \int_{\mathbf{R}}\left(1-\chi_{L}\right) u v \bar{\Phi}^{2} d x-2 \gamma \operatorname{Im} \int_{\mathbf{R}} v\left(1-\chi_{L}\right)\left[\int_{-\infty}^{x} u \bar{\Phi}+\Phi \bar{v} d x\right] \bar{\Phi} d x \\
& \leq 2 \eta\left\|w_{x}\right\|\left\|\Phi_{x}\right\|+2 \eta\|\Phi\|^{2}+2 \gamma \eta(\|u\|+\|v\|)\|\Phi\|^{2} .
\end{aligned}
$$


Let $\eta$ small sufficiently, such that $2 \eta[1+\gamma(\|u\|+\|v\|)] \leq \alpha$, then $\frac{d}{d t}\|\Phi\|^{2}+\alpha\|\Phi\|^{2} \leq C \eta$. By Gronwall inequality

$$
\|\Phi\|^{2} \leq\left\|u_{0}-v_{0}\right\|^{2} e^{-\alpha t}+\frac{C \eta}{\alpha}\left(1-e^{-\alpha t}\right), \quad t \geq 0 .
$$

On the other hand, multiplying (3.6) by $\bar{\Phi}_{x x}$, taking the imaginary part, and integrating on $\mathbf{R}$, we obtain

$$
\begin{aligned}
& \frac{d}{d t}\left\|\Phi_{x}\right\|^{2}+2 \eta\left\|\Phi_{x x}\right\|^{2}+2 \alpha\left\|\Phi_{x}\right\|^{2}=-2 \operatorname{Im} \int_{\mathbf{R}} i \eta w_{x x} \bar{\Phi}_{x x} d x-2 \operatorname{Im} \int_{\mathbf{R}} u v\left(1-\chi_{L}\right) \bar{\Phi}_{x x} d x \\
& -2 \operatorname{Im} \int_{\mathbf{R}}\left(|u|^{2}+|v|^{2}\right)\left(1-\chi_{L}\right) \Phi \bar{\Phi}_{x x} d x-2 \operatorname{Im} \int_{\mathbf{R}} \gamma \Phi\left[\left(1-\chi_{L}\right) \int_{-\infty}^{x}|u|^{2} d x\right] \bar{\Phi}_{x x} d x \\
& -2 \operatorname{Im} \int_{\mathbf{R}} \gamma v\left(1-\chi_{L}\right)\left[\int_{-\infty}^{x}(u \bar{\Phi}+\Phi \bar{v}) d x\right] \bar{\Phi}_{x x} d x \leq C \eta\left\|\Phi_{x x}\right\| .
\end{aligned}
$$

Thus, $\frac{d}{d t}\left\|\Phi_{x}\right\|^{2}+\alpha\left\|\Phi_{x}\right\|^{2} \leq C \eta$, by Gronwall inequality

$$
\left\|\Phi_{x}\right\|^{2} \leq\left\|u_{0 x}-v_{0 x}\right\|^{2} e^{-\alpha t}+\frac{C \eta}{\alpha}\left(1-e^{-\alpha t}\right), \quad t \geq 0 .
$$

Thus the proof of Lemma 3.5 is completed.

Lemma 3.6 There exists a constant $k_{2}>0$ such that, for any $\eta \in(0,1)$,

$$
\|\Psi\|_{H^{2}(\mathbf{R})} \leq k_{2}, t \geq 0
$$

Proof. Multiplying (3.8) by $\bar{\Psi}$, taking the imaginary part, and integrating on $\mathbf{R}$, we obtain

$$
\begin{aligned}
& \frac{d}{d t}\|\Psi\|^{2}+2 \eta\left\|\Psi_{x}\right\|^{2}+2 \alpha\|\Psi\|^{2}=-2 \operatorname{Im} \int_{\mathbf{R}} u v \bar{\Psi}^{2} d x-2 \operatorname{Im} \int_{\mathbf{R}} u v \chi_{L} \overline{\Phi \Psi} d x \\
& -2 \gamma \operatorname{Im} \int_{\mathbf{R}} v\left[\int_{-\infty}^{x}(u \bar{\Psi}+\Psi \bar{v}) d x\right] \bar{\Psi} d x-2 \gamma \operatorname{Im} \int_{\mathbf{R}} \Phi\left[\chi_{L} \int_{-\infty}^{x}|u|^{2} d x\right] \bar{\Psi} d x \\
& -2 \gamma \operatorname{Im} \int_{\mathbf{R}} v \chi_{L}\left[\int_{-\infty}^{x}(u \bar{\Phi}+\Phi \bar{v}) d x\right] \bar{\Psi} d x \leq C\|\Psi\|^{2} .
\end{aligned}
$$

Then by Gronwall inequality,

$$
\|\Psi\|^{2} \leq e^{C t}, \quad t \geq 0
$$

Multiplying (3.8) by $\bar{\Psi}_{x x}$, taking the imaginary part, and integrating on $\mathbf{R}$, we obtain

$$
\begin{aligned}
& \frac{d}{d t}\left\|\Psi_{x}\right\|^{2}+2 \eta\left\|\Psi_{x x}\right\|^{2}+2 \alpha\left\|\Psi_{x}\right\|^{2}=-2 \operatorname{Im} \int_{\mathbf{R}}\left(|u|^{2}+|v|^{2}\right) \Psi \bar{\Psi}_{x x} d x \\
& -2 \operatorname{Im} \int_{\mathbf{R}} u v \bar{\Psi}_{x x} d x-2 \gamma \operatorname{Im} \int_{\mathbf{R}} \Psi\left[\int_{-\infty}^{x}|u|^{2} d x\right] \bar{\Psi}_{x x} d x \\
& -2 \gamma \operatorname{Im} \int_{\mathbf{R}} v\left[\int_{-\infty}^{x}(u \bar{\Psi}+\Psi \bar{v}) d x\right] \bar{\Psi}_{x x} d x-2 \operatorname{Im} \int_{\mathbf{R}}\left(|u|^{2}+|v|^{2}\right) \chi_{L} \Phi \bar{\Psi}_{x x} d x \\
& -2 \operatorname{Im} \int_{\mathbf{R}} u v \chi_{L} \overline{\Phi \Psi}_{x x} d x-2 \gamma \operatorname{Im} \int_{\mathbf{R}} \Phi \chi_{L}\left[\int_{-\infty}^{x}|u|^{2} d x\right] \bar{\Psi}_{x x} d x \\
& -2 \gamma \operatorname{Im} \int_{\mathbf{R}} v \chi_{L}\left[\int_{-\infty}^{x}(u \bar{\Phi}+\Phi \bar{v}) d x\right] \bar{\Psi}_{x x} d x \leq C+\eta\left\|\Psi_{x x}\right\|^{2} .
\end{aligned}
$$

Thus, $\frac{d}{d t}\left\|\Psi_{x}\right\|^{2}+\alpha\left\|\Psi_{x}\right\|^{2} \leq C$, by Gronwall inequality

$$
\left\|\Psi_{x}\right\|^{2} \leq \frac{C}{\alpha}\left(1-e^{-\alpha t}\right), \quad t \geq 0 .
$$


Now multiplying (3.8) by $\bar{\Psi}_{x x x x}$, taking the imaginary part, and integrating on $\mathbf{R}$, we obtain

$$
\begin{aligned}
& \frac{d}{d t}\left\|\Psi_{x x}\right\|^{2}+2 \eta\left\|\Psi_{x x x}\right\|^{2}+2 \alpha\left\|\Psi_{x x}\right\|^{2}=2 \operatorname{Im} \int_{\mathbf{R}}\left(\left(|u|^{2}+|v|^{2}\right) \Psi\right)_{x} \bar{\Psi}_{x x x} d x \\
& +2 \operatorname{Im} \int_{\mathbf{R}}(u v \bar{\Psi})_{x} \bar{\Psi}_{x x x} d x+2 \gamma \operatorname{Im} \int_{\mathbf{R}}\left(\Psi\left[\int_{-\infty}^{x}|u|^{2} d x\right]\right)_{x} \bar{\Psi}_{x x x} d x \\
& +2 \gamma \operatorname{Im} \int_{\mathbf{R}}\left(v\left[\int_{-\infty}^{x}(u \bar{\Psi}+\Psi \bar{v}) d x\right]\right)_{x} \bar{\Psi}_{x x x} d x+2 \operatorname{Im} \int_{\mathbf{R}}\left(\left(|u|^{2}+|v|^{2}\right) \chi_{L} \Phi\right)_{x} \bar{\Psi}_{x x x} d x \\
& +2 \gamma \operatorname{Im} \int_{\mathbf{R}}\left(\Phi \chi_{L}\left[\int_{-\infty}^{x}|u|^{2} d x\right]\right)_{x} \bar{\Psi}_{x x x} d x+2 \operatorname{Im} \int_{\mathbf{R}}\left(u v \chi_{L} \bar{\Phi}\right)_{x} \bar{\Psi}_{x x x} d x \\
& +2 \gamma \operatorname{Im} \int_{\mathbf{R}}\left(v \chi_{L}\left[\int_{-\infty}^{x}(u \bar{\Phi}+\Phi \bar{v}) d x\right]\right)_{x} \bar{\Psi}_{x x x} d x \leq C+\eta\left\|\Psi_{x x x}\right\|^{2} .
\end{aligned}
$$

Thus, $\frac{d}{d t}\left\|\Psi_{x x}\right\|^{2}+\alpha\left\|\Psi_{x x}\right\|^{2} \leq C$, by Gronwall inequality

$$
\left\|\Psi_{x x}\right\|^{2} \leq \frac{C}{\alpha}\left(1-e^{-\alpha t}\right), \quad t \geq 0 .
$$

Thus the proof of Lemma 3.6 is completed.

Lemma 3.7 There exists a constant $k_{3}>0$ such that, for any $\eta \in(0,1)$,

$$
\|x \Psi\|_{H^{1}(\mathbf{R})} \leq k_{3}, t \geq 0
$$

Proof. Multiplying (3.8) by $x^{2} \bar{\Psi}$, taking the imaginary part, and integrating on $\mathbf{R}$, we obtain

$$
\begin{aligned}
& \frac{d}{d t}\|x \Psi\|^{2}+2 \eta\left\|\Psi_{x}\right\|^{2}+2 \alpha\|x \Psi\|^{2}=-2 \gamma \operatorname{Im} \int_{\mathbf{R}} v\left[\int_{-\infty}^{x}(u \bar{\Psi}+\Psi \bar{v}) d x\right] x^{2} \bar{\Psi} d x \\
& -2 \operatorname{Im} \int_{\mathbf{R}} u v x^{2} \bar{\Psi}^{2} d x-2 \operatorname{Im} \int_{\mathbf{R}}\left(|u|^{2}+|v|^{2}\right) \chi_{L} \Phi x^{2} \bar{\Psi} d x-2 \operatorname{Im} \int_{\mathbf{R}} u v \chi_{L} \bar{\Phi} x^{2} \bar{\Psi} d x \\
& -2 \gamma \operatorname{Im} \int_{\mathbf{R}} \Phi \chi_{L}\left[\int_{-\infty}^{x}|u|^{2} d x\right] x^{2} \bar{\Psi} d x-2 \gamma \operatorname{Im} \int_{\mathbf{R}} v \chi_{L}\left[\int_{-\infty}^{x}(u \bar{\Phi}+\Phi \bar{v}) d x\right] x^{2} \bar{\Psi} d x .
\end{aligned}
$$

Majorizing each term of the right hand side of (3.18), we have $\frac{d}{d t}\|x \Psi\|^{2} \leq C\|x \Psi\|^{2}+C$. By Gronwall inequality, we get $\|x \Psi\|^{2} \leq C$. Now let us differentiate (3.8) with respect to $x$, this leads to

$$
\begin{aligned}
& i \Psi_{x t}+(1-i \eta) \Psi_{x x x}+i \alpha \Psi_{x}+\left(|u|^{2}+|v|^{2}\right)_{x} \Psi+\left(|u|^{2}+|v|^{2}\right) \Psi_{x}+(u v)_{x} \bar{\Psi} \\
& +(u v) \bar{\Psi}_{x}+\gamma \Psi|u|^{2}+\gamma \Psi_{x} \int_{-\infty}^{x}|u|^{2} d x+\gamma v(u \bar{\Psi}+\Psi \bar{v})+\gamma v_{x} \int_{-\infty}^{x}(u \bar{\Psi}+\Psi \bar{v}) d x \\
& +\left[\left(|u|^{2}+|v|^{2}\right) \chi_{L} \Phi\right]_{x}+\left[u v \chi_{L} \bar{\Phi}\right]_{x}+\gamma\left(\Phi \chi_{L}\right)_{x} \int_{-\infty}^{x}|u|^{2} d x+\gamma \Phi \chi_{L}|u|^{2} \\
& +\gamma v \chi_{L}(u \bar{\Phi}+\Phi \bar{v})+\gamma\left(v \chi_{L}\right)_{x} \int_{-\infty}^{x}(u \bar{\Phi}+\Phi \bar{v}) d x=0, \quad t>0, x \in \mathbf{R} .
\end{aligned}
$$


Multiplying (3.19) by $x^{2} \bar{\Psi}_{x}$, taking the imaginary part, and integrating on $\mathbf{R}$, we obtain

$$
\begin{aligned}
& \frac{d}{d t}\left\|x \Psi_{x}\right\|^{2}+2 \eta\left\|x \Psi_{x x}\right\|^{2}+2 \alpha\left\|x \Psi_{x}\right\|^{2}=-2 \operatorname{Im} \int_{\mathbf{R}} x^{2}\left(|u|^{2}+|v|^{2}\right) \Psi \bar{\Psi}_{x} d x \\
& -2 \operatorname{Im} \int_{\mathbf{R}} x^{2}(u v)_{x} \bar{\Psi}_{x} d x-2 \operatorname{Im} \int_{\mathbf{R}} x^{2}(u v) \bar{\Psi}_{x}^{2} d x-2 \gamma \operatorname{Im} \int_{\mathbf{R}} x^{2}|u|^{2} \Psi \bar{\Psi}_{x} d x \\
& -2 \gamma \operatorname{Im} \int_{\mathbf{R}} x^{2}(u \bar{\Psi}+\Psi \bar{v}) \bar{\Psi}_{x} d x-2 \gamma \operatorname{Im} \int_{\mathbf{R}} x^{2} v_{x}\left[\int_{-\infty}^{x}(u \bar{\Psi}+\Psi \bar{v}) d x\right] \bar{\Psi}_{x} d x \\
& -2 \operatorname{Im} \int_{\mathbf{R}} x^{2}\left[\left(|u|^{2}+|v|^{2}\right) \chi_{L} \Phi\right]_{x} \bar{\Psi}_{x} d x-2 \operatorname{Im} \int_{\mathbf{R}} x^{2}\left[u v \chi_{L} \bar{\Phi}\right]_{x} \bar{\Psi}_{x} d x \\
& -2 \gamma \operatorname{Im} \int_{\mathbf{R}} x^{2} \Phi \chi_{L}|u|^{2} \bar{\Psi}_{x} d x-2 \gamma \operatorname{Im} \int_{\mathbf{R}} x^{2}\left(\Phi \chi_{L}\right)_{x}\left[\int_{-\infty}^{x}|u|^{2} d x\right] \bar{\Psi}_{x} d x \\
& -2 \gamma \operatorname{Im} \int_{\mathbf{R}} x^{2} v \chi_{L}(u \bar{\Phi}+\Phi \bar{v}) \bar{\Psi}_{x} d x-2 \gamma \operatorname{Im} \int_{\mathbf{R}} x^{2}\left(v \chi_{L}\right)_{x}\left[\int_{-\infty}^{x}(u \bar{\Phi}+\Phi \bar{v}) d x\right] \bar{\Psi}_{x} d x .
\end{aligned}
$$

Majorizing each term of the right hand side of (3.20), we have $\frac{d}{d t}\left\|x \Psi_{x}\right\|^{2} \leq C\left\|x \Psi_{x}\right\|^{2}+C$. By Gronwall inequality, we get $\left\|x \Psi_{x}\right\|^{2} \leq C$. Thus the proof of Lemma 3.7 is completed.

Proof of Lemma 3.4. From (3.10), for all $\delta \in\left(0, \frac{1}{4}\right)$, let $t$ large enough such that $\|\Phi\|_{H^{1}(\mathbf{R})} \leq$ $\frac{\delta}{8}\|w(0)\|_{H^{1}(\mathbf{R})}$. Let

$$
B_{0}=\left\{\Psi \quad \mid\|\Psi\|_{H^{2}(\mathbf{R})}^{2}+\left\|x \Psi_{x}\right\|^{2} \leq k_{2}^{2}+k_{3}^{2}=K^{2}\right\} .
$$

Note that the left-hand side of the inequality in (3.21) can be written in the following form:

$$
\|\Psi\|_{H^{2}(\mathbf{R})}^{2}+\left\|x \Psi_{x}\right\|^{2}=(L \Psi, \Psi), \quad L=-\Psi_{x x}+\Psi+x^{2} \Psi .
$$

Thanks to Lemma 3.5, $B_{0}$ is compact subset in $H^{1}(\mathbf{R})$, hence the operator $L^{-1}$ is compact. Since the left-hand side of (3.21) is a quadratic function of $\Psi$, it defines an ellipsoid $B_{0}$ in $H^{1}(\mathbf{R})$, and since it is compact, it may be written as $B_{0}=\left\{\Psi \mid \sum_{j=1}^{\infty}\left(\Psi, e_{j}\right)^{2} \lambda_{j} \leq K^{2}\right\}$, where $\left\{e_{j}\right\}_{j=1}^{\infty}$ is an complete orthonormal basis in $H^{1}(\mathbf{R}), e_{j}$ is an eigenfunction of operator $L, \lambda_{j}$ is eigenvalue of the operator $L$ corresponding with $e_{j}, 0<\lambda_{1}<\lambda_{2}<\cdots<\lambda_{m}<\cdots \rightarrow+\infty$, as $j \rightarrow+\infty$. Let $N$ be so large that $\lambda_{N} \geq \frac{16 K^{2}}{\delta^{2}\|w(0)\|^{2}}$. Let $E_{N}=\left\{e_{1}, e_{2}, \cdots, e_{N}\right\}, P_{N}: H^{1}(\mathbf{R}) \rightarrow E_{N}$, the orthoprojector onto $E_{N}$. Therefore, if $\Psi \in B_{0}$, then

$$
\left\|\left(I-P_{N}\right) \Psi\right\|^{2}=\sum_{j=N+1}^{\infty}\left(\Psi, e_{j}\right)^{2} \leq \frac{K^{2}}{\lambda_{N}} \leq \frac{\delta^{2}}{16 K^{2}}\|w(0)\|^{2} .
$$

Thus

$$
\left\|\left(I-P_{N}\right) w\right\|^{2} \leq\left\|\left(I-P_{N}\right) \Phi\right\|^{2}+\left\|\left(I-P_{N}\right) \Psi\right\|^{2} \leq\|\Phi\|^{2}+\left\|\left(I-P_{N}\right) \Psi\right\|^{2} \leq \frac{3 \delta}{8}\|w(0)\|^{2} .
$$

Therefore, if $\left\|\left(I-P_{N}\right) w\right\| \leq\left\|P_{N} w\right\|,\|w\| \leq 2\left\|\left(I-P_{N}\right) w\right\| \leq \frac{3 \delta}{4}\|w(0)\|^{2}<\delta\|w(0)\|^{2}$. Thus the proof of Lemma 3.4 is completed.

\section{Proof of Theorem 1.3}

In this section, we shall prove the regularity of global attractor for the problems (1.1)-(1.2). To this end, let $u$ be the solution of (1.1)-(1.2) which also reads $u(x)=\frac{1}{2 \pi} \int_{\mathbf{R}} \widehat{u}(\xi) e^{i x \xi} d \xi$. On one hand, for a given level $N$, we denote by $y$ the low frequency part of $u$ as $y(x)=\frac{1}{2 \pi} \int_{|\xi| \leq N} \widehat{u}(\xi) e^{i x \xi} d \xi$. We note that $y$ is a smooth function with respect to the $x$ variable. On the other hand, we also define the high frequency 
part of $u$ as $z(x)=\frac{1}{2 \pi} \int_{|\xi|>N} \widehat{u}(\xi) e^{i x \xi} d \xi$. We observe that the regularity of $u$ with respect to $x$ depends on its high frequency part. By projecting (1.1) on the high modes, we see that $z$ is the solution of the non-autonomous partial differential equation

$$
\begin{array}{r}
i z_{t}+z_{x x}+Q\left[\gamma(y+z)\left(\int_{-\infty}^{x}|y+z|^{2} d x\right)\right]+Q\left[|y+z|^{2}(y+z)\right]+i \alpha z=Q f \\
z(0)=Q u_{0}=z_{0}
\end{array}
$$

where $Q$ denotes the orthogonal projector onto $Q H^{1}=\left\{z \in H^{1}(\mathbf{R}) \quad \mid z=\frac{1}{2 \pi} \int_{|\xi|>N} \widehat{u}(\xi) e^{i x \xi} d \xi\right\}$. Since we are interested in the long time behavior of $z(t)$, we may focus on $z(t)$ for $t \geq t_{0}$, $t_{0}$ being as in Lemma 2.2. Hence, for $t \geq t_{0}, z$ is the solution of (4.1) with the following initial condition $z\left(t_{0}\right)=Q u\left(t_{0}\right)$. We also introduce $Z:\left[t_{0},+\infty\right) \rightarrow Q H^{1}$ which is the solution of

$$
i Z_{t}+Z_{x x}+Q\left[\gamma(y+Z) \int_{-\infty}^{x}|y+Z|^{2} d x\right]+Q\left[|y+Z|^{2}(y+Z)\right]+i \alpha Z=Q f,
$$

with $Z\left(t_{0}\right)=0$, here $y=(I-Q) u$ is as above. The existence result for $Z$ will be proven in the following Lemma 4.1. Moreover, in Lemma 4.2, we shall prove that for a given $N$ large enough, depending on $\alpha$ and $f, Z(t)$ exists for all $t \geq t_{0}$ and takes its values in $Q H^{2}$. Thus we split $z$ into two parts as follows $z=Z+(z-Z)$, here $Z$ is smooth, and $z-Z$ converges towards 0 in $H^{1}(\mathbf{R})$ when $t \rightarrow+\infty$. For the sake of convenience, in this section, we assume that $t_{0}=0$, that is

$$
Z(0)=0
$$

Hence $u(t)$ remains in the absorbing ball in $H^{1}(\mathbf{R})$ whose radius is $\rho$ as in (2.4). We state and prove the following result:

Lemma 4.1 There exists $N_{0}$ that depends on the data of the equation $\alpha$ and $f$, such that for a given $N \geq N_{0}, Z(t) \in C_{b}\left((0,+\infty), Q H^{1}\right)$ is the solution of (4.3)-(4.4). Moreover, we have

$$
\sup _{t \geq 0}\|Z(t)\|_{H^{1}(\mathbf{R})} \leq K_{1} .
$$

Proof. Let $N$ be fixed large enough, $m>N$ and $y(t)=(I-Q) u(t)$ as above. Assume that $Z^{m}(t)$ be the solution in $P_{m} Q H^{1}=\left\{Z^{m} \mid Z^{m}=\frac{1}{2 \pi} \int_{N<|\xi|<m} \widehat{u}(\xi) e^{i x \xi} d \xi\right\}$ of the equation

$$
i Z_{t}^{m}+Z_{x x}^{m}+P_{m} Q\left[\gamma\left(y+Z^{m}\right) \int_{-\infty}^{x}\left|y+Z^{m}\right|^{2}\right]+P_{m} Q\left[\left|y+Z^{m}\right|^{2}\left(y+Z^{m}\right)\right]+i \alpha Z^{m}=P_{m} Q f
$$

with $Z^{m}(0)=0$. This equation is a Galerkin approximation at order $m$ of problem (4.3), we shall prove the global existence of $Z^{m}$ and we obtain the existence of $Z$ by letting $m \rightarrow \infty$. Due to Cauchy-Lipschitz theorem, $Z^{m}$ exists in $\left[0, T_{m}\right)$ and $Z^{m} \in P_{m} Q H^{1}$ for some $T_{m}$. Thus we only need to prove below a priori estimates which show the fact $T_{m}=+\infty$ and which allow us to let $m$ go to infinity:

$$
\sup _{t \geq 0}\left\|Z^{m}(t)\right\|_{H^{1}(\mathbf{R})} \leq K_{1}
$$

For the sake of convenience, we drop the superscript $m$ and write $Z=Z^{m}, v=v^{m}=y+Z^{m}$. To prove (4.7), let us take the imaginary part of the scalar product of (4.6) with $Z$, and taking the real part of the scalar product of (4.6) with $-Z_{t}-\alpha Z-i \gamma(y+Z) \int_{-\infty}^{x}|y+Z|^{2} d x$, we obtain

$$
\frac{1}{2} \frac{d}{d t} J_{1}(Z)+\alpha J_{1}(Z)=J_{2}(Z)
$$

where

$$
\left.J_{1}(Z)=\|Z\|_{H^{1}(\mathbf{R})}^{2}+\int_{\mathbf{R}} \operatorname{Re}\left(-2 \bar{Z}|y|^{2} y\right)-\frac{1}{2}|Z|^{4}-2|Z|^{2}|y|^{2}-\bar{Z}^{2} y^{2}-2|Z|^{2} \bar{Z} y+2 \bar{Z} f\right) d x,
$$




$$
\begin{aligned}
J_{2}(Z)= & \int_{\mathbf{R}} \operatorname{Im}\left(\bar{Z} f-\bar{Z}|y|^{2} y-|Z|^{2} Z \bar{y}-\bar{Z}^{2} y^{2}-2|Z|^{2} \bar{Z} y\right) d x \\
& +\alpha \int_{\mathbf{R}} \operatorname{Re}\left(\frac{1}{2}|Z|^{4}-\bar{Z}|y|^{2} y+|Z|^{2} \bar{Z} y+\bar{Z} f\right) d x \\
& -\int_{\mathbf{R}} \operatorname{Re}\left(|Z|^{2}\left(|y|^{2}\right)_{t}+\bar{Z}\left(|y|^{2} y\right)_{t}+\bar{Z}^{2} y y_{t}+|Z|^{2} \bar{Z} y_{t}\right) d x \\
& +\gamma \int_{\mathbf{R}} \operatorname{Im}\left(Z_{x}(\overline{y+Z})|y+Z|^{2}+\left(\bar{y}_{x} Z_{x}+\bar{y}_{x} f+\bar{Z} f-y \bar{Z}\right) \int_{-\infty}^{x}|y+Z|^{2} d x\right) d x .
\end{aligned}
$$

We now majorize $J_{1}(Z)$.

$$
\begin{aligned}
J_{1}(Z) \geq & \|Z\|_{H^{1}(\mathbf{R})}^{2}-2\|Z\|_{L^{4}(\mathbf{R})}\|y\|_{L^{4}(\mathbf{R})}^{3}-3\|Z\|_{L^{4}(\mathbf{R})}^{2}\|y\|_{L^{4}(\mathbf{R})}^{2} \\
& -\frac{1}{2}\|Z\|_{L^{4}(\mathbf{R})}^{4}-2\|Z\|_{L^{4}(\mathbf{R})}^{3}\|y\|_{L^{4}(\mathbf{R})}-2\|Z\|_{L^{4}(\mathbf{R})}\|f\|_{L^{\frac{4}{3}}(\mathbf{R})} .
\end{aligned}
$$

Due to Sobolev embedding $H^{1}(\mathbf{R}) \subset L^{4}(\mathbf{R})$ and to the fact that $y=(I-Q) u$, we have

$$
\|y\|_{L^{4}(\mathbf{R})} \leq C\|y\|_{H^{1}(\mathbf{R})} \leq C\|u\|_{H^{1}(\mathbf{R})} \leq C \rho,
$$

where $\rho$ is in (2.4). Thus we obtain

$$
J_{1}(Z) \geq\|Z\|_{H^{1}(\mathbf{R})}^{2}-\|Z\|_{L^{4}(\mathbf{R})}^{4}-K .
$$

We now majorize $J_{2}(Z)$. Firstly, we have

$$
\begin{aligned}
& \int_{\mathbf{R}} \operatorname{Im}\left(\bar{Z} f-\bar{Z}|y|^{2} y-|Z|^{2} Z \bar{y}-\bar{Z}^{2} y^{2}-2|Z|^{2} \bar{Z} y\right) d x \\
& +\alpha \int_{\mathbf{R}} \operatorname{Re}\left(\frac{1}{2}|Z|^{4}-\bar{Z}|y|^{2} y+|Z|^{2} \bar{Z} y+\bar{Z} f\right) d x \\
\leq & (1+\alpha)\|Z\|_{L^{4}(\mathbf{R})}\|y\|_{L^{4}(\mathbf{R})}^{3}+\|Z\|_{L^{4}(\mathbf{R})}^{2}\|y\|_{L^{4}(\mathbf{R})}^{2}+\frac{\alpha}{2}\|Z\|_{L^{4}(\mathbf{R})}^{4} \\
& +(3+\alpha)\|Z\|_{L^{4}(\mathbf{R})}^{3}\|y\|_{L^{4}(\mathbf{R})}+(1+\alpha)\|Z\|_{L^{4}(\mathbf{R})}\|f\|_{L^{\frac{4}{3}(\mathbf{R})}} \\
\leq & \alpha\|Z\|_{L^{4}(\mathbf{R})}^{4}+K .
\end{aligned}
$$

Secondly, using the enhanced Poincaré inequality that holds on $Q H^{1},\|Z\|_{L^{\infty}(\mathbf{R})} \leq \frac{C}{\sqrt{N}}\|Z\|_{H^{1}(\mathbf{R})}$, we get

$$
\begin{aligned}
& -\int_{\mathbf{R}} \operatorname{Re}\left(|Z|^{2}\left(|y|^{2}\right)_{t}+\bar{Z}\left(|y|^{2} y\right)_{t}+\bar{Z}^{2} y y_{t}+|Z|^{2} \bar{Z} y_{t}\right) d x \\
\leq & C\|y\|_{L^{\infty}(\mathbf{R})}\|Z\|_{L^{\infty}(\mathbf{R})}\|Z\|_{H^{1}(\mathbf{R})}+C\|y\|_{H^{1}(\mathbf{R})}\|Z\|_{L^{\infty}(\mathbf{R})}^{2} \\
& +C\|y\|_{H^{1}(\mathbf{R})}\|y\|_{L^{\infty}(\mathbf{R})}\|Z\|_{L^{\infty}(\mathbf{R})}+C\|y\|_{L^{\infty}(\mathbf{R})}^{2}\|Z\|_{H^{1}(\mathbf{R})} \\
& +C\|Z\|_{L^{\infty}(\mathbf{R})}^{2}\|Z\|_{H^{1}(\mathbf{R})} \\
\leq & \frac{C}{\sqrt{N}}\|Z\|_{H^{1}(\mathbf{R})}^{2}+\frac{\alpha}{4}\|Z\|_{H^{1}(\mathbf{R})}^{2}+\frac{C}{N}\|Z\|_{H^{1}(\mathbf{R})}^{3}+K .
\end{aligned}
$$

Lastly, we get easily the following four inequalities:

$$
\begin{aligned}
& \gamma \int_{\mathbf{R}} \operatorname{Im}\left(Z_{x}(\overline{y+Z})|y+Z|^{2}\right) d x \\
\leq & C\|Z\|_{H^{1}(\mathbf{R})}+\frac{C}{\sqrt{N}}\|Z\|_{H^{1}(\mathbf{R})}^{2}+\frac{C}{N}\|Z\|_{H^{1}(\mathbf{R})}^{3}+\frac{C}{N}\|Z\|_{H^{1}(\mathbf{R})}^{4}, \\
& \gamma \int_{\mathbf{R}} \operatorname{Im}\left(\bar{y}_{x} Z_{x} \int_{-\infty}^{x}|y+Z|^{2} d x\right) d x \\
\leq & \mid \gamma\left\|y_{x}\right\|\left\|Z_{x}\right\|\left(\|y\|_{L^{\infty}(\mathbf{R})}^{2}+2\|y\|_{L^{\infty}(\mathbf{R})}\|Z\|_{L^{\infty}(\mathbf{R})}+\|Z\|_{L^{\infty}(\mathbf{R})}^{2}\right) \\
\leq & C\|Z\|_{H^{1}(\mathbf{R})}+\frac{C}{\sqrt{N}}\|Z\|_{H^{1}(\mathbf{R})}^{2}+\frac{C}{N}\|Z\|_{H^{1}(\mathbf{R})}^{3},
\end{aligned}
$$




$$
\begin{aligned}
& \gamma \int_{\mathbf{R}} \operatorname{Im}\left((\overline{y+Z}) f \int_{-\infty}^{x}|y+Z|^{2} d x\right) d x \\
\leq & |\gamma| \int_{\mathbf{R}}\left(|y|^{3}+3|y|^{2}|Z|+3|y||Z|^{2}+|Z|^{3}\right)|f| d x \leq C\|Z\|_{H^{1}(\mathbf{R})}^{3}+K, \\
& -\gamma \int_{\mathbf{R}} \operatorname{Im}\left(y \bar{Z} \int_{-\infty}^{x}|y+Z|^{2} d x\right) d x \leq C\|Z\|_{H^{1}(\mathbf{R})}+C\|Z\|_{H^{1}(\mathbf{R})}^{2}+\frac{C}{N}\|Z\|_{H^{1}(\mathbf{R})}^{3} .
\end{aligned}
$$

From above inequalities we obtain

$$
J_{2}(Z) \leq\left(\frac{\alpha}{4}+\frac{K}{\sqrt{N}}\right)\|Z\|_{H^{1}(\mathbf{R})}^{2}+\alpha\|Z\|_{L^{4}(\mathbf{R})}^{4}+\frac{K^{\prime}}{N}\|Z\|_{H^{1}(\mathbf{R})}^{3}+K^{\prime \prime} .
$$

Hence, by (4.8) and (4.10), we get

$$
\frac{1}{2} \frac{d}{d t} J_{1}(Z)+\alpha J_{1}(Z) \leq \frac{3 \alpha}{8}\|Z\|_{H^{1}(\mathbf{R})}^{2}+\frac{K}{\sqrt{N}}\|Z\|_{H^{1}(\mathbf{R})}^{2}+\frac{K^{\prime}}{N^{2}}\|Z\|_{H^{1}(\mathbf{R})}^{4}+K^{\prime \prime},
$$

where $K, K^{\prime}, K^{\prime \prime}$ are constants. Let us assume that

$$
\frac{K}{\sqrt{N}} \leq \frac{K}{\sqrt{N_{0}}} \leq \frac{\alpha}{8}, \quad \text { i.e. } \quad N_{0} \geq \frac{64 K^{2}}{\alpha^{2}},
$$

then it follows from (4.9) and (4.10) that

$$
\frac{d}{d t} J_{1}(Z)+\alpha J_{1}(Z) \leq \frac{K}{N^{2}}\|Z\|_{H^{1}(\mathbf{R})}^{4}+K^{\prime} .
$$

Integrating (4.13) for $t$ between 0 and $t$ (observing that $J_{1}(Z(0))=J_{1}(0)=0$ ), we have

$$
J_{1}(Z(t)) e^{\alpha t} \leq \frac{K}{N^{2}} \int_{0}^{t}\|Z(s)\|_{H^{1}(\mathbf{R})}^{4} e^{\alpha s} d s+\frac{K^{\prime} e^{\alpha t}}{\alpha} .
$$

Thus we deduce easily from (4.9) that

$$
\|Z\|_{H^{1}}^{2} \leq \frac{C}{N^{3}}\|Z\|_{H^{1}(\mathbf{R})}^{4}+\frac{K}{N^{2}} \int_{0}^{t}\|Z(s)\|_{H^{1}(\mathbf{R})}^{4} e^{\alpha(s-t)} d s+K^{\prime} .
$$

Now let us introduce $\zeta(t)=\sup _{[0, t]}\|Z(s)\|_{H^{1}(\mathbf{R})}^{2}$, which is a continuous function of $t$ satisfying $\zeta(0)=0$. By (4.15), $\zeta(t)$ satisfies $\zeta(t) \leq \frac{K}{N^{2}} \zeta^{2}(t)+K^{\prime}$. Set $\phi(\zeta)=\zeta-\frac{K}{N^{2}} \zeta^{2}-K^{\prime}$, and assume that $\phi(2 K)>0$, namely $\frac{4 K K^{\prime}}{N^{2}} \leq \frac{4 K K^{\prime}}{N_{0}^{2}}<1$, i.e., $N_{0} \geq 2 \sqrt{K K^{\prime}}$. Since $\phi(\zeta(0))=\phi(0)<0$ and $t \rightarrow \zeta(t)$ is a continuous nonnegative function of $t$, then $\zeta(t)$ remains bounded in $\left[0, \alpha_{1}\right]$, where $\alpha_{1}$ is the first root of $\phi$. Thus we have $\zeta(t)=\sup _{[0, t]}\|Z(s)\|_{H^{1}(\mathbf{R})}^{2} \leq 2 K^{\prime}$, which is independent of $m$, the proof of Lemma 4.1 is completed.

Lemma 4.2 Let $N_{0}$ be as in Lemma 4.1. Let $N \geq N_{0}$ be fixed. There exists $K(N)$ that depends on $N$, such that the solution $Z$ of (4.3)-(4.4) satisfies

$$
\sup _{t \geq 0}\|Z(t)\|_{H^{2}(\mathbf{R})} \leq K(N) .
$$

Proof. Let us take the imaginary part of the scalar product of (4.6) with $Z_{x x}^{m}$. Here for the sake of convenience, we drop the superscript $m$ and write $Z=Z^{m}, v=v^{m}=y+Z^{m}$. This leads to

$$
\frac{1}{2} \frac{d}{d t}\left\|Z_{x}\right\|^{2}+\alpha\left\|Z_{x}\right\|^{2}=\int_{\mathbf{R}} \operatorname{Im}\left(G \bar{Z}_{x}-v^{2} \bar{Z}_{x}^{2}-\gamma|v|^{2} Z \bar{Z}_{x}\right) d x,
$$

where

$$
G=P_{m} Q\left[f_{x}-(v)^{2} \bar{y}_{x}-2|v|^{2} y_{x}-\gamma\left(\int_{-\infty}^{x}|v|^{2} d x\right) y_{x}-\gamma|v|^{2} y\right]
$$


Taking the real part of the scalar product of (4.6) with $-Z_{x x t}-\alpha Z_{x x}-i \gamma Z_{x} \int_{-\infty}^{x}|v|^{2} d x$, we obtain

$$
\begin{aligned}
& \frac{1}{2} \frac{d}{d t}\left\{\left\|Z_{x x}\right\|^{2}+\int_{\mathbf{R}} \operatorname{Re}\left(2 G \bar{Z}_{x}-\bar{Z}_{x}^{2} v^{2}-2\left|Z_{x}\right|^{2}|v|^{2}-2 \gamma|v|^{2} Z \bar{Z}_{x}\right) d x\right\} \\
& +\alpha\left\{\left\|Z_{x x}\right\|^{2}+\int_{\mathbf{R}} \operatorname{Re}\left(G \bar{Z}_{x}-\bar{Z}_{x}^{2} v^{2}-2\left|Z_{x}\right|^{2}|v|^{2}-\gamma|v|^{2} Z \bar{Z}_{x}\right) d x\right\} \\
& =\int_{\mathbf{R}} \operatorname{Re}\left(G_{t} \bar{Z}_{x}-v v_{t} \bar{Z}_{x}^{2}-2\left|Z_{x}\right|^{2} v v_{t}-\gamma|v|^{2} Z_{t} \bar{Z}_{x}\right) d x \\
& \quad-2 \gamma \int_{\mathbf{R}} \operatorname{Re}\left(v \bar{v}_{t}\right) \operatorname{Re}\left(Z \bar{Z}_{x}\right) d x-\gamma \int_{\mathbf{R}} \operatorname{Im}\left(|v|^{2} Z_{x x} \bar{Z}_{x}\right) d x \\
& \quad+\gamma \int_{\mathbf{R}} \operatorname{Im}\left(\left(G \bar{Z}_{x}-Z_{x}^{2} v^{2}-\gamma|v|^{2} Z \bar{Z}_{x}\right) \int_{-\infty}^{x}|v|^{2} d x\right) d x .
\end{aligned}
$$

It follows from (4.17)-(4.19) that

$$
\frac{1}{2} \frac{d}{d t} J_{3}\left(Z_{x}\right)+\alpha J_{3}\left(Z_{x}\right)=J_{4}\left(Z_{x}\right)
$$

where

$$
\begin{aligned}
& J_{3}\left(Z_{x}\right)=\left\|Z_{x}\right\|_{H^{1}(\mathbf{R})}^{2}+\int_{\mathbf{R}} \operatorname{Re}\left(2 G \bar{Z}_{x}-\bar{Z}_{x}^{2} v^{2}-2\left|Z_{x}\right|^{2}|v|^{2}-2 \gamma|v|^{2} Z \bar{Z}_{x}\right) d x, \\
& \geq \frac{3}{4}\left\|Z_{x}\right\|_{H^{1}(\mathbf{R})}^{2}-2\|G\|_{H^{-1}(\mathbf{R})}\left\|Z_{x}\right\|_{H^{1}(\mathbf{R})}-2|\gamma| \int_{\mathbf{R}}|v|^{2}|Z|\left|Z_{x}\right| d x \\
& \geq \frac{1}{2}\left\|Z_{x}\right\|_{H^{1}(\mathbf{R})}^{2}-C\|G\|_{H^{-1}(\mathbf{R})}^{2} . \\
& J_{4}\left(Z_{x}\right)=-\gamma \int_{\mathbf{R}} \operatorname{Im}\left(Z_{x}^{2} v^{2} \int_{-\infty}^{x}|v|^{2} d x\right) d x-\gamma^{2} \int_{\mathbf{R}} \operatorname{Im}\left(|v|^{2} Z \bar{Z}_{x} \int_{-\infty}^{x}|v|^{2} d x\right) d x \\
& -\gamma \int_{\mathbf{R}} \operatorname{Im}\left(|v|^{2} Z_{x x} Z_{x}\right) d x-\gamma \int_{\mathbf{R}} \operatorname{Re}\left(|v|^{2} Z_{t} \bar{Z}_{x}\right) d x-\int_{\mathbf{R}} \operatorname{Im}\left(\bar{Z}_{x}^{2} v^{2}\right) d x \\
& -\gamma \int_{\mathbf{R}} \alpha \operatorname{Re}\left(|v|^{2} Z \bar{Z}_{x}\right)+\operatorname{Im}\left(|v|^{2} Z \bar{Z}_{x}\right) d x+\int_{\mathbf{R}} \operatorname{Re}\left(-v v_{t} \bar{Z}_{x}^{2}-2\left|Z_{x}\right|^{2} v v_{t}\right) d x \\
& -2 \gamma \int_{\mathbf{R}} \operatorname{Re}\left(v \bar{v}_{t}\right) \operatorname{Re}\left(Z \bar{Z}_{x}\right) d x+\gamma \int_{\mathbf{R}} \operatorname{Im}\left(G \bar{Z}_{x} \int_{-\infty}^{x}|v|^{2} d x\right) d x \\
& +\int_{\mathbf{R}} \alpha \operatorname{Re}\left(G \bar{Z}_{x}\right)+\operatorname{Im}\left(G \bar{Z}_{x}\right) d x+\int_{\mathbf{R}} \operatorname{Re}\left(G_{t} \bar{Z}_{x}\right) d x . \\
& \leq \frac{C K_{0}^{4}}{\sqrt{N}}\left\|Z_{x}\right\|_{H^{1}(\mathbf{R})}^{2}+\frac{C K_{0}^{2}}{\sqrt{N}}\left\|Z_{x}\right\|_{H^{1}(\mathbf{R})}^{2}+\frac{C K_{0}^{2}}{\sqrt{N}}\|G\|_{H^{-1}(\mathbf{R})}\left\|Z_{x}\right\|_{H^{1}(\mathbf{R})} \\
& +(\alpha+1)\|G\|_{H^{-1}(\mathbf{R})}\left\|Z_{x}\right\|_{H^{1}(\mathbf{R})}+\left\|G_{t}\right\|_{H^{-1}(\mathbf{R})}\left\|Z_{x}\right\|_{H^{1}(\mathbf{R})} \\
& \leq\left(\frac{C K_{0}^{2}}{\sqrt{N}}+\frac{C K_{0}^{4}}{\sqrt{N}}+\frac{\alpha}{8}\right)\left\|Z_{x}\right\|_{H^{1}(\mathbf{R})}^{2}+C\left(\left\|G_{t}\right\|_{H^{-1}(\mathbf{R})}^{2}+\|G\|_{H^{-1}(\mathbf{R})}^{2}\right) \text {. }
\end{aligned}
$$

Assuming that $N \geq N_{2}$ is large enough to satisfy $\frac{C K_{0}^{2}}{\sqrt{N}}+\frac{C K_{0}^{4}}{\sqrt{N}} \leq \frac{C K_{0}^{2}}{\sqrt{N_{2}}}+\frac{C K_{0}^{4}}{\sqrt{N_{2}}} \leq \frac{\alpha}{8}$, i.e. $N_{2} \geq \frac{64 C^{2} K_{0}^{4}\left(1+K_{0}^{2}\right)^{2}}{\alpha}$, we get

$$
\frac{1}{2} \frac{d}{d t} J_{3}\left(Z_{x}\right)+\frac{\alpha}{2} J_{3}\left(Z_{x}\right) \leq C\left(\|G\|_{H^{-1}(\mathbf{R})}^{2}+\left\|G_{t}\right\|_{H^{-1}(\mathbf{R})}\right) .
$$

By Gronwall lemma and by $J_{3}\left(Z_{x}(0)\right)=J_{3}(0)=0$, we obtain the following inequality

$$
\sup _{t \geq 0}\left\|Z_{x}^{m}(t)\right\|_{H^{1}(\mathbf{R})} \leq \frac{C}{\alpha} \sup _{t \geq 0}\left(\|G(t)\|_{H^{-1}(\mathbf{R})}^{2}+\left\|G_{t}(t)\right\|_{H^{-1}(\mathbf{R})}\right) .
$$


Now we estimate the right hand side of (4.24). Applying the following inverse inequality $\left\|y_{x}\right\|_{H^{k}(\mathbf{R})} \leq$ $(2 \pi N)^{k-1}\|y\|_{H^{1}(\mathbf{R})}$, we have the following inequality

$$
\begin{aligned}
\sup _{t \geq 0}\|G\|_{H^{1}(\mathbf{R})} & \leq \sup _{t \geq 0}\left[\left\|f_{x}\right\|_{H^{1}(\mathbf{R})}+C\left\|y_{x}\right\|_{H^{1}(\mathbf{R})}\left\|v^{m}\right\|_{H^{1}}^{2}+C\|y\|_{H^{1}(\mathbf{R})}\left\|v^{m}\right\|_{H^{1}(\mathbf{R})}^{2}\right] \\
& \leq \sup _{t \geq 0}\left[\left\|f_{x}\right\|_{H^{1}(\mathbf{R})}+C\|y\|_{H^{1}(\mathbf{R})}\left\|v^{m}\right\|_{H^{1}(\mathbf{R})}^{2}\right] \leq C .
\end{aligned}
$$

Hence we have bound $G$ in $C_{b}^{1}\left((0,+\infty), H^{-1}(\mathbf{R})\right)$. It remains to consider its time derivative. The derivative of $G$ reads:

$$
\begin{aligned}
G_{t}= & P_{m} Q\left[-4 \operatorname{Re}\left(\overline{v^{m}} v_{t}^{m}\right) y_{x}-2\left|v^{m}\right|^{2} y_{x t}-\left(v^{m}\right)^{2} \overline{y_{x t}}-2 v^{m} v_{t}^{m} \overline{y_{x}}-\gamma\left|v^{m}\right|^{2} y_{t}\right. \\
& \left.-2 \gamma\left(\int_{-\infty}^{x} \operatorname{Re}\left(\overline{v^{m}} v_{t}^{m}\right) d x\right) y_{x}-\gamma\left(\int_{-\infty}^{x}\left|v^{m}\right|^{2} d x\right) y_{x t}-2 \gamma \operatorname{Re}\left(\overline{v^{m}} v_{t}^{m}\right) y\right] .
\end{aligned}
$$

Firstly, let $\phi$ be a test function in $H^{1}(\mathbf{R})$ that satisfies $\|\phi\|_{H^{1}(\mathbf{R})}=1$, we have the following two inequalities

$$
\begin{aligned}
& \sup _{\|\phi\|_{H^{1}(\mathbf{R})}=1}\left(-4 \operatorname{Re}\left(\overline{v^{m}} v_{t}^{m}\right) y_{x}, \phi\right)_{L^{2}} \leq \sup _{\|\phi\|_{H^{1}(\mathbf{R})}=1} 4\left\|v_{t}^{m}\right\|_{H^{-1}(\mathbf{R})}\left\|\left|v^{m}\left\|y_{x}\right\| \phi\right|\right\|_{H^{1}(\mathbf{R})} \leq K(N), \\
& \sup _{\|\phi\|_{H^{1}(\mathbf{R})}=1}\left(-2 \gamma \int_{-\infty}^{x} \operatorname{Re}\left(\overline{v^{m}} v_{t}^{m}\right) d s y_{x}, \phi\right)_{L^{2}(\mathbf{R})} \leq K(N) .
\end{aligned}
$$

On the other hand, applying the inverse inequality $\left\|y_{x t}\right\|_{H^{-1}(\mathbf{R})} \leq(2 \pi N)^{2}\left\|y_{t}\right\|_{H^{-1}(\mathbf{R})}$, we get the following two inequalities

$$
\begin{aligned}
& \sup _{\|\phi\|_{H^{1}(\mathbf{R})}=1}\left(-\gamma \int_{-\infty}^{x}\left|v^{m}\right|^{2} d x y_{x t}, \phi\right)_{L^{2}(\mathbf{R})} \leq \sup _{\|\phi\|_{H^{1}(\mathbf{R})}=1}|\gamma|\left\|\left|v^{m}\right|^{2}|\phi|\right\|_{H^{1}}\left\|y_{x t}\right\|_{H^{-1}} \leq K(N), \\
& \sup _{\|\phi\|_{H^{1}(\mathbf{R})}=1}\left(-2\left|v^{m}\right|^{2} y_{x t}, \phi\right)_{L^{2}(\mathbf{R})} \leq K(N) .
\end{aligned}
$$

Similarly, we get the following four inequalities

$$
\begin{array}{r}
\sup _{\|\phi\|_{H^{1}(\mathbf{R})}=1}\left(-\left(v^{m}\right)^{2} \overline{y_{x t}}, \phi\right)_{L^{2}(\mathbf{R})} \leq K(N), \quad \sup _{\|\phi\|_{H^{1}(\mathbf{R})}=1}\left(-2 v^{m} v_{t}^{m} \overline{y_{x}}, \phi\right)_{L^{2}(\mathbf{R})} \leq K(N), \\
\sup _{\|\phi\|_{H^{1}(\mathbf{R})}=1}\left(-2 \gamma \operatorname{Re}\left(\overline{v^{m}} v_{t}^{m}\right) y, \phi\right)_{L^{2}(\mathbf{R})} \leq K(N), \quad \sup _{\|\phi\|_{H^{1}(\mathbf{R})}=1}\left(-\gamma\left|v^{m}\right|^{2} y_{t}, \phi\right)_{L^{2}(\mathbf{R})} \leq K(N) .
\end{array}
$$

Since the projector $Q$ is uniformly bounded from $H^{-1}(\mathbf{R})$ into $H^{-1}(\mathbf{R})$, then we obtain that $G_{t}$ is bounded in $C_{b}\left((0,+\infty), H^{-1}(\mathbf{R})\right)$.

By above inequalities and (4.24), we get (4.16), hence the proof of Lemma 4.2 is completed.

Now we aim to compare $z$ with $Z$ for large time. Let $z=Q u$ that solves (4.1) and let $Z$ solve (4.3), then we get the following result:

Lemma 4.3 Let $N_{0}$ be as in Lemma 4.1. For $N \geq N_{0}$, we have

$$
\|Z(t)-z(t)\|_{H^{1}(\mathbf{R})} \leq C_{0} e^{-\alpha t}
$$

where $C_{0}$ depends on $\left\|u_{0}\right\|_{H^{1}(\mathbf{R})}$.

Proof. Let $v=y+Z$ and $w=Z-z=v-u$. Thus $w$ satisfies

$$
i w_{t}+w_{x x}+i \alpha w+\gamma Q\left[w \int_{-\infty}^{x}|v|^{2} d x+u \int_{-\infty}^{x}(\bar{u} w+v \bar{w}) d x\right]+Q\left[\left(|u|^{2}+|v|^{2}\right) w+u v \bar{w}\right]=0
$$

Multiplying (4.26) by $\bar{w}$, taking the imaginary part, and multiplying (4.26) by $-\bar{w}_{t}-\alpha \bar{w}-i \gamma Q\left[\bar{w} \int_{-\infty}^{x}|v|^{2} d x+\bar{u} \int_{-\infty}^{x}(\bar{u} w+v \bar{w}) d x\right]$, taking the real part, we obtain

$$
\frac{1}{2} \frac{d}{d t} J_{5}(w)+\alpha J_{5}(w)=J_{6}(w)
$$


where

$$
\begin{aligned}
& J_{5}(w)=\|w\|_{H^{1}(\mathbf{R})}^{2}-\int_{\mathbf{R}}\left(|u|^{2}+|v|^{2}\right)|w|^{2}+\operatorname{Re}\left(u v \bar{w}^{2}\right) d x . \\
J_{6}(w)= & -\int_{\mathbf{R}} \operatorname{Im}\left(u v \bar{w}^{2}-\gamma u \bar{w} \int_{-\infty}^{x}(\bar{u} w+v \bar{w}) d x\right) d x \\
& +\gamma \int_{\mathbf{R}}\left[\alpha|w|^{2}-\operatorname{Im}\left(u v \bar{w}^{2}\right)\right] \int_{-\infty}^{x}|v|^{2} d x d x \\
& +\gamma \alpha \int_{\mathbf{R}} \operatorname{Re}\left((u \bar{w}-\bar{u} w) \int_{-\infty}^{x}(\bar{u} w+v \bar{w}) d x\right) d x \\
& -\frac{1}{2} \int_{\mathbf{R}}\left(\left(|u|^{2}+|v|^{2}\right)_{t}|w|^{2}+\operatorname{Re}\left((u v)_{t} \bar{w}^{2}\right)\right) d x \\
& -\gamma \int_{\mathbf{R}} \operatorname{Im}\left(w_{x} w|v|^{2}+w \bar{u}^{2} w+w \bar{u} v \bar{w}\right) d x \\
& -\gamma^{2} \int_{\mathbf{R}} \operatorname{Im}\left((u \bar{w}+\bar{u} w) \int_{-\infty}^{x}(\bar{u} w+v \bar{w}) d x \int_{-\infty}^{x}|v|^{2} d x\right) d x \\
& -\gamma \int_{\mathbf{R}} \operatorname{Im}\left(\left(|u|^{2} w \bar{u}+|v|^{2} w \bar{u}+|u|^{2} v \bar{w}+w_{x} \bar{u}_{x}\right) \int_{-\infty}^{x}(\bar{u} w+v \bar{w}) d x\right) d x . \\
& -\gamma^{2} \int_{\mathbf{R}} \operatorname{Im}\left(|u|^{2}\left[\int_{-\infty}^{x}(\bar{u} w+v \bar{w}) d x\right]^{2}\right) d x .
\end{aligned}
$$

Since $Z$ satisfies (4.4) and since $\|u\|_{H^{1}} \leq M_{1}$, we have

$$
J_{5}(w) \geq\|w\|_{H^{1}(\mathbf{R})}^{2}\left(1-\frac{C\left(M_{1}^{2}+K_{1}^{2}\right)}{N^{2}}\right) .
$$

Hence if $N_{0}$ is large enough as required by Lemma 4.1, we can conclude on the coerciveness of $J_{5}$ on $H^{1}$, that is,

$$
J_{5}(w) \geq \frac{1}{2}\|w\|_{H^{1}(\mathbf{R})}^{2} .
$$

Next we majorize the right hand side of (4.29). Firstly, we have

$$
\begin{aligned}
& -\int_{\mathbf{R}} \operatorname{Im}\left(u v \bar{w}^{2}-\gamma u \bar{w} \int_{-\infty}^{x}(\bar{u} w+v \bar{w}) d x\right) d x \\
& \leq\|w\|_{L^{\infty}(\mathbf{R})}^{2}\left(\|u\|\|v\|+\gamma\|u\|^{2}+\gamma\|u\|\|v\|\right) \leq \frac{K}{2 \sqrt{N}}\|w\|_{H^{1}(\mathbf{R})}^{2} .
\end{aligned}
$$

Majorize similarly the rest terms of the right hand side of (4.29), we have

$$
J_{6}(w) \leq \frac{K}{2 \sqrt{N}}\|w\|_{H^{1}(\mathbf{R})}^{2}
$$

Thus we easily imply from (4.27) and (4.31) that

$$
\frac{1}{2} \frac{d}{d t} J_{5}(w)+\alpha J_{5}(w) \leq \frac{K}{\sqrt{N}}\|w\|_{H^{1}(\mathbf{R})}^{2} \leq \frac{2 K}{\sqrt{N}} J_{5}(w) .
$$

Since $\frac{2 K}{\sqrt{N}} \leq \frac{\alpha}{2}$ (as in Lemma 4.1), the classical Gronwall lemma leads to

$$
J_{5}(w(t)) \leq J_{5}(w(0)) e^{-\alpha t}
$$

By using $w(0)=z_{0}$ and the coerciveness of $J$ on $Q H^{1}$, we easily complete the proof of (4.28). The proof of Lemma 4.3 is completed. 
Proof of Theorem 1.3. Lemma 4.2 implies that $\mathcal{A}_{\gamma}$ is a bounded subset of $H^{2}(\mathbf{R})$. We shall prove the compactness. We introduce an energy equation for $u$ which reads

$$
J_{7}\left(S_{\gamma}(t) u_{0}\right)=J_{7}\left(u_{0}\right) e^{-2 \alpha t}+\int_{0}^{t} e^{-2 \alpha(t-s)} J_{8}\left(S_{\gamma}(s) u_{0}\right) d s
$$

where

$$
\begin{aligned}
J_{7}(u)= & \left\|u_{x x}\right\|^{2}-\int_{\mathbf{R}} \operatorname{Re}\left(f \bar{u}_{x x}\right) d x-\int_{\mathbf{R}}\left[|u|^{2}\left|u_{x}\right|^{2}+2\left(\operatorname{Re}\left(u_{x} \bar{u}\right)\right)^{2}\right] d x \\
& -\gamma \int_{\mathbf{R}}\left[\left|u_{x}\right|^{2}\left(\int_{-\infty}^{x}|u|^{2} d x\right)+2|u|^{2} \operatorname{Re}\left(u_{x} \bar{u}\right)\right] d x \\
J_{8}(u)= & 2 \alpha\left\|u_{x x}\right\|^{2}-2 \alpha \int_{\mathbf{R}} \operatorname{Re}\left(f \bar{u}_{x x}\right) d x-2 \operatorname{Re} \alpha \int_{\mathbf{R}} \bar{u}_{x}\left(|u|^{2} u\right)_{x} d x \\
& -2 \operatorname{Re} \alpha \int_{\mathbf{R}} \bar{u}_{x}\left(u \int_{\infty}^{x}|u|^{2} d x\right)_{x} d x,+\gamma \int_{\mathbf{R}}\left[\left|u_{x}\right|^{2} \int_{\infty}^{x}\left(|u|^{2}\right)_{t} d x\right. \\
& \left.+2\left(|u|^{2}\right)_{t} \operatorname{Re}\left(u_{x} \bar{u}\right)+2|u|^{2} \operatorname{Re}\left(u_{x} \bar{u}_{t}\right)\right] d x \\
& +\int_{\mathbf{R}}\left[\left|u_{x}\right|^{2}\left(|u|^{2}\right)_{t}+4 \operatorname{Re}\left(u_{x} \bar{u}\right) \operatorname{Re}\left(u_{x} \bar{u}_{t}\right)\right] d x .
\end{aligned}
$$

Let $x_{j}$ be a sequence in $\mathcal{A}_{\gamma}$. Since $\mathcal{A}_{\gamma}$ is bounded in $H^{2}(\mathbf{R})$, there exists a subsequence $x_{j}$, that weakly converges towards $\xi$ in $H^{2}(\mathbf{R})$, and strongly in $H^{1}(\mathbf{R})$, because $\mathcal{A}_{\gamma}$ is compact in $H^{1}(\mathbf{R})$. For fixed nonnegative $t$, let $u_{0}=S(-t) x_{j^{\prime}}$, we have

$$
\limsup _{j^{\prime} \rightarrow+\infty} J_{7}\left(x_{j^{\prime}}\right) \leq J_{7}(\xi)-J_{7}(S(-t) \xi) e^{-\alpha t}+\limsup _{j^{\prime} \rightarrow+\infty} J_{7}\left(S(-t) x_{j^{\prime}}\right) e^{-\alpha t} .
$$

As $J_{7}$ is bounded on $\mathcal{A}_{\gamma}$, then when $t \rightarrow \infty, \limsup _{j^{\prime} \rightarrow+\infty}\left\|x_{j^{\prime}}\right\|_{H^{2}(\mathbf{R})} \leq\|\xi\|_{H^{2}(\mathbf{R})}$. We then obtain the result.

\section{Proof of Theorem 1.4}

In this section our main goal is to prove the Theorem 1.4. It is equivalent to proving the following statement: let $y=P u_{1}(t)=P u_{2}(t), P=P_{N}$, such that

$$
\|y\|_{H^{1}(\mathbf{R})} \leq \rho_{1}, \quad\left\|y_{t}\right\|_{H^{-1}(\mathbf{R})} \leq \rho_{2}, \quad t \in \mathbf{R} .
$$

Then there exists only one solution $z=z(t) \in C_{b}\left(\mathbf{R}, Q H^{1}\right)$ of the nonlinear autonomous equation

$$
\begin{array}{r}
i z_{t}+z_{x x}+Q\left[\gamma(y+z)\left(\int_{-\infty}^{x}|y+z|^{2} d x\right)\right]+Q\left[|y+z|^{2}(y+z)\right]+i \alpha z=Q f \\
\limsup _{t \rightarrow-\infty}\|z\|_{H^{1}(\mathbf{R})}<+\infty
\end{array}
$$

Let $z_{1}(t)$ and $z_{2}(t)$ be two solutions of (5.2)-(5.3), we now follow step by step the proof of Lemma 4.3. We set $v_{1}=y+z_{1}$ and $v_{2}=y+z_{2}, w=z_{2}-z_{1}=v_{2}-v_{1}$. Then $w$ satisfies

$$
\begin{aligned}
i w_{t}+w_{x x}+\gamma Q\left[w \int_{-\infty}^{x}\left|v_{2}\right|^{2} d x+v_{1} \int_{-\infty}^{x}\left(\bar{v}_{2} w+v_{1} \bar{w}\right) d x\right] \\
+Q\left[\left(\left|v_{1}\right|^{2}+\left|v_{2}\right|^{2}\right) w+v_{1} v_{2} \bar{w}\right]+i \alpha w=0 .
\end{aligned}
$$

Multiplying (5.4) by $\bar{w}$, taking the imaginary part, and multiplying (5.4) by $-\bar{w}_{t}-\alpha \bar{w}-i \gamma Q\left[\bar{w} \int_{-\infty}^{x}|v|^{2} d x+\bar{u} \int_{-\infty}^{x}(\bar{u} w+v \bar{w}) d x\right]$, taking the real part, we obtain, as in (4.35),

$$
\frac{1}{2} \frac{d}{d t} J_{9}(w)+\alpha J_{9}(w) \leq 0
$$


where

$$
J_{9}(w)=\|w\|_{H^{1}(\mathbf{R})}^{2}-\int_{\mathbf{R}}\left(\left|v_{1}\right|^{2}+\left|v_{2}\right|^{2}\right)|w|^{2}+\operatorname{Re}\left(v_{1} v_{2} \bar{w}^{2}\right) d x \geq \frac{1}{2}\|w\|_{H^{1}(\mathbf{R})}^{2},
$$

provided $N \geq N_{0}$. Integrating (5.5) between $t^{\prime}$ and $t>t^{\prime}$, and also using (5.6), we have

$$
\|w\|_{H^{1}(\mathbf{R})}^{2} \leq J_{9}\left(w\left(t^{\prime}\right)\right) e^{-\alpha\left(t-t^{\prime}\right)} \quad \text { for } t \geq t^{\prime} .
$$

We now let $t^{\prime}$ go to $-\infty$. Observing that $J_{9}\left(w\left(t^{\prime}\right)\right)$ remains bounded, thus we have proved the Theorem 1.4 .

\section{Proof of Theorem 1.5}

In this section our main goal is to prove the Theorem 1.5. Assume that $u, v$ are the solutions of the nonlocal and usual Schrödinger equations, respectively, with the same initial data $u_{0}(x)$, i.e., $u=S_{\gamma}(t) u_{0}$, $v=S(t) u_{0}$. We denote also $w=u-v$, and hence $w(0)=0$. Using the equations (1.1) and (1.3), we get

$$
i w_{t}+w_{x x}+|u|^{2} w+u v \bar{w}+|v|^{2} w+\gamma u \int_{-\infty}^{x}|u|^{2} d x+i \alpha w=0 .
$$

Multiplying (6.1) by $\bar{w}$, taking the imaginary part, we obtain

$$
\begin{aligned}
\frac{d}{d t}\|w\|^{2}+2 \alpha\|w\|^{2} & =-2 \operatorname{Im} \int_{\mathbf{R}} u v \bar{w}^{2} d x-2 \gamma \operatorname{Im} \int_{\mathbf{R}} u \bar{w}\left[\int_{-\infty}^{x}|u|^{2} d x\right] d x \\
& \leq 2\|u\|_{L^{\infty}}\|v\|_{L^{\infty}}\|w\|^{2}+2 \gamma\|u\|^{3}\|w\| \leq C_{1}\|w\|^{2}+C_{2} \gamma^{2} .
\end{aligned}
$$

Thus, since $w(0)=0$, we have

$$
\|w(t)\|^{2} \leq \frac{C_{2}}{C_{1}}\left(e^{C_{1} t}-1\right) \gamma^{2}
$$

Multiplying (6.1) by $-\bar{w}_{x x}$, taking the imaginary part, we obtain

$$
\begin{aligned}
\frac{1}{2} \frac{d}{d t}\left\|w_{x}\right\|^{2}+\alpha\left\|w_{x}\right\|^{2}= & -\operatorname{Im} \int_{\mathbf{R}}\left(|u|^{2}+|v|^{2}\right)_{x} w \bar{w}_{x} d x-\operatorname{Im} \int_{\mathbf{R}}(u v)_{x} \overline{w w}_{x} d x \\
& -\operatorname{Im} \int_{\mathbf{R}} u v \bar{w}_{x}^{2} d x-\gamma \operatorname{Im} \int_{\mathbf{R}} u_{x} \bar{w}_{x}\left[\int_{-\infty}^{x}|u|^{2} d x\right] d x \\
& -\gamma \operatorname{Im} \int_{\mathbf{R}} u \bar{w}_{x}|u|^{2} d x \leq C_{3}\left\|w_{x}\right\|^{2}+C_{4} \gamma^{2}
\end{aligned}
$$

Then

$$
\left\|w_{x}(t)\right\|^{2} \leq \frac{C_{4}}{C_{3}}\left(e^{C_{3} t}-1\right) \gamma^{2}
$$

By (6.2)-(6.3), we conclude that $\|w(t)\|_{H^{1}(\mathbf{R})}^{2} \rightarrow 0$ as $\gamma \rightarrow 0$. This shows that the solution $u$ of the nonlocal Schrödinger equation (1.1) approaches the solution $v$ of the usual Schrödinger equation (1.3). Moreover, solution operator $S_{\gamma}(t)$ has a uniform domain of attraction, i.e., $H^{1}(\mathbf{R})$, which is independent of $\gamma$. Thus we have proved the Theorem 1.5.

\section{Acknowledgments}

This work was supported by the National Natural Sciences Foundation of China (No.11571283) and the Postdoctoral Research Fundation of Chongqing (No. yuXM201102006). 


\section{References}

1. F. Abergel, "Attractors for a Navier-Stokes ow in an unbounded domain," Math. Mod. Num. Anal., vol. 23, no. 3, pp. 359-370, 1989.

2. F. Abergel, "Existence and finite dimensionality of the global attractor for evolution equations on unbounded domains," J. Diff. Equs., vol. 83, no. 1, pp. 85-108, 1990.

3. A. V. Babin, "The attractor of a Navier-Stokes system in an unbounded channel-like domain," J. Dyna. Diff. Equs., vol. 4, no. 4, pp. 555-584, 1992.

4. A. V. Babin, "Global attractors in PDE," in: B. Hasselblatt and A. K. atok (Ed.), Handbook of Dynamical Systems, vol. 1B, Elsevier, pp. 983-1085, 2006.

5. A. V. Babin, B. Nicolaenko, "Exponential attractors of Reaction-Diffusion Systems in an unbounded domain," J. Dyna. Diff. Equs., vol. 7, no. 4, pp. 567-590, 1995.

6. A. V. Babin, M. I. Vishik, "Attractors of partial differential equations in an unbounded domain," Proc. Roy. Soc. Edinburgh, vol. 116A, pp. 221-243, 1990.

7. A. V. Babin, M. I. Vishik, "Attractors of Evolutionary Equations," North-Holland, 1989.

8. T. Cazenave, A. Haraux, "Introduction aux problèmes d'évoluion semilinéaires, Ellipses," Pairs, 1990.

9. A. Eden, C. Foias, B. Nicolaenko, Z. S. Shen, "Exponential attractors and their relevance to fluid dynamics systems," Physica D, vol. 63, no. 3-4, pp. 350-360, 1993.

10. A. Eden, C. Foias, B. Nicolaenko, R. Temam, "Inertial sets for dissipative evolution equations," Part I: Construction and applications, IMA Preprint Series 812, University Minnesota, 1991.

11. E. Feireisl, P. Laurençot, F. Simondon, and H. Touré. Compact attractors for reaction-diffusion equations in $\mathbf{R}^{N}, "$ C. R. Acad. Sci. Paris, Série I, vol. 319, no. 2, pp. 147-151, 1994.

12. E. Feireisl, "Attractors for semilinear damped wave equations on $\mathbf{R}^{3}$," Nonlin. Anal., vol. 23, no. 2, pp. 187-195, 1994.

13. C. Foias, O. Manley, R. Temam and Y. Treve, "Asymptotic analysis of the Navier-Stokes equations," Physica $D$, vol. 9, no. 1-2, pp. 157-188, 1983.

14. C. Foias, G. Prodi, "Sur le comportement global des solutions non stationnaires des ëquations de Navier-Stokes en dimension 2," Rend. Sem. Mat. Univ. padova, vol. 39, no. 1, pp. 1-34, 1967.

15. J. M. Ghidaglia, Finite dimensional behavior for the weakly damped driven Schrödinger equation," Ann. I. H. Poincare, vol. 5, no. 4, pp. 365-405, 1988.

16. J. M. Ghidaglia, R. Temam, "Regularity of the solutions of second order evolution equations and their attractors," Ann. Sc. Norm. Super. Pisa Cl. Sci. IV, vol. 14, no. 4, pp. 485-511, 1987.

17. O. Goubet, "Regularity of the attractor for a weakly damped Schrödinger equations," Appl. Anal., vol. 60, no. 1-2, pp. 99-119, 1996

18. O. Goubet, "Asymptotic smoothing effect for a weakly damped nonlinear Schröinger equation in $T^{2}, " J$. Differ. Equs., vol. 165, no. 1, pp. 96-122, 2000.

19. O. Goubet, I. Moise, "Attractors for dissipative Zakharov system," Nonl. Anal. TMA, vol. 31, no. 1, pp. $823-847,1988$

20. B. L. Guo, Y. S. Li, "Attractor for Dissipative Klein-Gordon-Schrödinger Equations in $\mathbf{R}^{3}$," J. Diff. Equs., vol. 136, no. 2, pp. 356-377, 1997.

21. J. K. Hale, "Smoothing properties of neutral equations," An. Acad. Brasil. Cienc., vol. 45, no. 1, pp. 49-50, 1973.

22. J. K. Hale, "Asymptotic Behavior of Dissipative Systems," Math. Surveys and Monographs, vol. 25, Amer. Math. Soc., Providence, RI, 1988.

23. J. K. Hale, G. Raugel, "Regularity, determining modes and Galerkin methods," J. Math. Pures Appl., vol. 82, no. 9, pp. 1075-1136, 2003.

24. J. K. Hale, G. Raugel, "A modified Poincaré method for the persistence of periodic orbits and applications," J. Dyna. Diff. Equs., vol. 22, no. 1, pp. 3-68, 2010.

25. J. K. Hale, G. Raugel, "Persistence of periodic orbits for perturbed dissipative dynamical systems," Infinite Dimensional Dynamical Systems Fields Institute Communications, vol. 64, pp. 1-55, 2013.

26. J. K. Hale, X. B. Lin, G. Raugel, "Upper semicontinuity of attractors for approximations of semigroups and partial differential equations," Math. Comput., vol. 50, no. 2, pp. 89-123, 1988.

27. D. Henry, "Geometric Theory of Semilinear Parabolic Equations," Lecture Notes in Math., vol. 840, SpringerVerlag, 1981.

28. A. T. Hill, E. Sulin, "Set convergence for discretization of the attractor," IMA J. Num. Analy., vol. 16, no. 2, pp. 289-296, 1996.

29. H. Y. Huang, "The finite Dimension Behavior of the Schrödinger Equation with Nonlocal Integral Nonlinearity," Acta. Math. Appl., vol. 15, no. 1, pp. 34-44, 1999.

30. Y. H. Ichikava, "Topics on Solitons in Plasmas," Physica Scripta, vol. 20, no. 3-4, pp. 296-305, 1979. 
31. P. Laurencot, "Long-time behaviour for weakly damped driven nonlinear Schrödinger equations in $\mathbf{R}^{N}$," $N \leq 3, N o D E A$, vol. 2, no. 3, 357-369, 1995.

32. O. Lopes, "Asymptotic fixed point theorems and forced oscillations in neutral equations," PhD thesis, Brown University, Providence, RI, June, 1973.

33. S. Q. Ma, Q. S. Chang, "Attractor and dimensions for discretizations of a NLS eauation with a non-local nonlinear term," Acta Math. Sinica, vol. 18, no. 4, pp. 779-800, 2002.

34. A. Miranville, S. Zelik, "Attractors for dissipative partial differential equations in bounded and unbounded domains," in C. M. Dafermos and M. Pokorny (Ed.), Handbook of Diff. Equs.: Evolut. Equs., vol. 4, Elsevier, pp. 103-200, 2008.

35. I. Moise, R. Rosa, "On the regularity of the global attractor of a weakly damped forced Korteweg-De-Vrie equation," Adv. Diff. Equs., vol. 2, no. 2, pp. 257-296, 1997.

36. I. Moise, R. Rosa, X. Wang, "Attractors for non-compact semigroups via energy equations," Nonlinearity, vol. 11, no. 5, pp. 1369-1393, 1998.

37. R. Nussbaum, "Periodic solutions of analytic functional differential equations are analytic," Michigan Math. J., vol. 20, no. 3, pp. 249-255, 1973.

38. M. Oliver, E. S. Titi, "Analyticity of the attractor and the number of determine nodes for a weakly damped driven nonlinear Schrödinger equation," Indiana Univ. Math. J., vol. 47, no. 1, pp. 47-73, 1998.

39. M. Paicu, G. Raugel, A. Rekaloc, "Regularity of the global attractor and finite-dimensional behavior for the second grade fluid equations," J. Diff. Equs., vol. 252, no. 6, pp. 3695-3751, 2012.

40. A. Pazy, "Semigroups of Linear Operators and Applications to Partial Differential Equations," Springer, 1983.

41. G. Raugel, "Global attractors in partial differential equations," in: B. Fiedler (Ed.), Handbook of Dynamical Systems, vol. 2, North-Holland, pp. 885-982, 2002.

42. R. Temam, "Infinite-Dimensional Dynamical Systems in Mechanics and Physics" (Second Ed), Springer-Verlag, New York, 1997.

43. B. X. Wang, "Regularity of attractors for the Benjamin-Bona-Mahony equation," J. Phys. A: Math. Gen. , vol. 31, no. 37, pp. 7635-7645, 1998.

44. X. M. Wang, "An energy equation for the weakly damped driven nonlinear Schrödinger equations and its applications to their attractors," Physic. D, vol. 88, no. 3-4, pp. 167-175, 1995.

45. C. K. Zhong, M. H. Yang, C. Y. Sun, "The existence of global attractors for the norm-to-weak continuous semigroup and application to the nonlinear reactionĺCdiffusion equations," J. Diff. Equus., vol. 223, no. 2, pp. 367-399, 2006.

46. C. S. Zhu, "Global attractor for the damped Benjamin-Bona-Mahony equations on $\mathbf{R}^{1}$," Appl. Anal., vol. 86, no. 1, pp. 59-65, 2007.

47. C. S. Zhu, C. L. Mu, Z. L. Pu, "Attractor for the Nonlinear Schrödinger Equation with a Non-local Nonlinear Term," J. Dyna. Contr. Syst., vol. 16, no. 3, pp. 585-603, 2010. 